Research Article

\title{
Robust Waveform Design Based on Bisection and Maximum Marginal Allocation Methods with the Concept of Information Entropy
}

\author{
Bin Wang $\mathbb{D D}^{1}$ and Xiaolei Hao $\mathbb{D D}^{2}$ \\ ${ }^{1}$ School of Electrical and Electronic Engineering, Shijiazhuang Tiedao University, Shijiazhuang, China \\ ${ }^{2}$ School of Computer and Communication Engineering, Northeastern University at Qinhuangdao, Qinhuangdao, China \\ Correspondence should be addressed to Bin Wang; wangbinneu@qq.com
}

Received 20 May 2020; Accepted 16 July 2020; Published 6 August 2020

Guest Editor: Wenguang Yu

Copyright (c) 2020 Bin Wang and Xiaolei Hao. This is an open access article distributed under the Creative Commons Attribution License, which permits unrestricted use, distribution, and reproduction in any medium, provided the original work is properly cited.

Cognitive radar can overcome the shortcomings of traditional radars that are difficult to adapt to complex environments and adaptively adjust the transmitted waveform through closed-loop feedback. The optimization design of the transmitted waveform is a very important issue in the research of cognitive radar. Most of the previous studies on waveform design assume that the prior information of the target spectrum is completely known, but actually the target in the real scene is uncertain. In order to simulate this situation, this paper uses a robust waveform design scheme based on signal-to-interference-plus-noise ratio (SINR) and mutual information (MI). After setting up the signal model, the SINR and MI between target and echo are derived based on the information theory, and robust models for MI and SINR are established. Next, the MI and SINR are maximized by using the maximum marginal allocation (MMA) algorithm and the water-filling method which is improved by bisection algorithm. Simulation results show that, under the most unfavorable conditions, the robust transmitted waveform has better performance than other waveforms in the improvement degree of SINR and MI. By comparing the robust transmitted waveform based on SINR criterion and MI criterion, the influence on the variation trend of SINR and MI is explored, and the range of critical value of $T_{y}$ is found. The longer the echo observation time is, the better the performance of the SINR-based transmitted waveform over the MIbased transmitted waveform is. For the mutual information between the target and the echo, the performance of the MMA algorithm is better than the improved water-filling algorithm.

\section{Introduction}

Radar uses radio method to find targets and determine their spatial position. However, with the wide application of electromagnetic spectrum, the working environment of radar is more and more complex. The traditional radar has a single transmitting waveform, which is difficult to adapt to the complex and changeable working environment. Cognitive radar is an intelligent radar system concept proposed in recent years. This system can improve the system performance of the radar through using the feedback structure from the receiver to the transmitter to optimize the transmitted waveform based on the recognition of the target and the scene. The whole system forms a closed-loop structure [1]. In view of the leading role of cognitive radar research in the development direction of radar, experts and scholars in various countries have launched research in related fields.

Adaptive waveform design is the key problem in cognitive radar research, which makes cognitive radar transmit the waveform that adapts to the change of environment. In the past decades, many experts and scholars devoted themselves to researching on transmitted waveform to improve the detection and estimation performance of radar system for extended target. In [2], based on information theory, the author proposes a water-filling algorithm to maximize the mutual information between the received radar waveform and the target. The author studies the use of information theory to design the waveform to measure the resonance phenomenon of the extended radar target. For the deterministic target impulse response and the random target 
impulse response, the radar waveform design problem with waveform energy and duration constraints is solved. The optimal target detection scheme puts as much energy as possible in the maximum target scattering mode to maximize the mutual information between target and the received radar echo. In [3], the authors propose a minimax robust signal processing scheme when the prior knowledge is inaccurate and discuss robust linear filters for signal estimation and signal detection. Related applications and nonlinear methods for robust signal detection and robust estimation are also studied. In [4], considering the uncertainty of the prior information of the radar target in the actual scene, the authors propose a waveform design method based on mutual information to ensure the parameter estimation performance of complex target models. This algorithm is robust to the uncertainty under the layered game model of radar and jammer and can effectively guarantee the parameter estimation performance. In [5], the authors study the relationship between the transmitted waveform and the multitarget mutual information in the two cases of noise only and clutter included according to the maximum mutual information criterion. Compared with the LFM signal, the waveform designed based on the maximum mutual information criterion can make the radar echo contain more information about multiple targets. In [6], the authors derive the convergence of the iterative water-filling algorithm and propose an algorithm that can guarantee its convergence in the presence of various forms of time-varying errors. Simulation results show that under the condition of strong interference, the traditional iterative water-filling algorithm is divergent, but the algorithm in this paper is still convergent. In [7], the authors focus on the transmitted waveform and filter structure of polarimetric radar. The worst-case signal-to-interference-plus-noise ratio is used as the criterion under both a similarity and an energy constraint on the transmit signal. An iterative optimization method for robust design is proposed. In [8], the authors propose a comprehensive theory of matched illumination waveforms for determining extended targets and random extended targets, use signal-to-noise ratio and mutual information as optimization criteria to design matched waveforms, and extensively discuss the waveform design of random targets and known targets with correlated interference based on SNR and MI. In [9], the authors propose a multitarget detection method and adaptive waveform design algorithm for MIMO cognitive radar, which models multitarget detection as multiple hypothesis testing. An adaptive waveform design algorithm based on information theory is proposed. The semidefinite relaxation technique and semidefinite programming are used to solve nonconvex design problems, which improves the efficiency of multiple hypothesis testing. In [10], the authors propose an adaptive orthogonal frequency division multiplexing radar communication waveform design method to improve the efficiency of limited spectrum resources and study the optimization problem of the conditional mutual information between the random target impulse response and the received signal and data information rate for frequency-selective fading channels. In [11], the author investigates the design of orthogonal frequency division multiplexing multiple-input multipleoutput radar waveforms with target uncertainty and improves the space-time adaptive processing detection performance of MIMO-OFDM radar in the most unfavorable case. The author proposes a method based on diagonal loading. By using the DL method, the optimization problem can be reduced to a semidefinite programming problem. In [12], the authors propose a robust waveform technique for multistatic cognitive radars in the context of signal-related clutter and derive a new method that directly assumes uncertainty on the radar cross section and Doppler parameter of the clutters. A specific clutter random optimization method using Taylor series approximation is proposed to determine a robust waveform with specific SINR outage constraints. In [13], the authors study the robust waveform design of multiple-input multiple-output cognitive radar and propose a two-step process. First, the covariance matrix of the detection signal is designed. Then, a waveform is synthesized from the obtained covariance matrix. In [14], the authors investigate the design of anglerobust joint transmit waveforms and receive filters for multiple-input multiple-output (MIMO) radars under signal-dependent interference. The method maximizes the output signal-to-interference-plus-noise ratio (SINR) in the most unfavorable case in unknown target angles. Based on rank-relaxed semidefinite programming (SDP) of nonnegative triangular polynomials, a cyclic optimization algorithm is proposed to solve this problem. In [15], the authors extend the traditional Gaussian target response to arbitrary nonGaussian target distributions, use cognitive radar multiple hypothesis classification algorithms for non-Gaussian targets, and utilize the sparse spectrum of related narrowband target responses. In previous studies, most of them assume that the target spectrum is known. However, the real target spectrum cannot be accurately captured in practice. Even if some researchers consider the uncertainty of target spectrum and use robust technology, the solution process is very complex.

In this paper, we fully consider the uncertainty of the target in practice. Based on the concept of entropy in information theory, we establish robust waveform design model of MI and SINR. Then, we use water-filling method improved by bisection algorithm and maximum marginal allocation algorithm to maximize MI and SINR. Finally, we obtain robust waveforms with better performance than other waveforms. The whole paper is organized as follows. Section 2 is the signal model. Section 3 is the robust waveform design based on MI. Section 4 is the robust waveform design based on SINR. Section 5 gives the search method of Lagrange multipliers based on bisection algorithm. Section 6 introduces the maximum marginal allocation algorithm. Section 7 shows the simulation results and related analysis. Section 8 concludes the whole paper.

\section{Signal Model}

Assume that the target model is a stationary random process on time interval $\left[0, T_{h}\right]$, with a value 0 outside of $\left[0, T_{h}\right]$. A stationary random process is a random process whose 
probability distribution at a fixed time and position is the same as that of all times and positions, which means the statistical characteristics of the random process do not change over time. $g(t)$ is a generalized stationary Gaussian random process, whose mathematical expectation and variance are independent of time, and its correlation function is only related to time interval. $a(t)$ is the rectangle window function, and the duration of the window function is $T_{h}$. Therefore, $h(t)=g(t) a(t)$ can be constructed, which is a random process with finite duration. Since $g(t)$ is generalized stationary, $h(t)$ is locally stationary in $\left[0, T_{h}\right]$.

$x(t)$ and $h(t)$ represent the transmitted waveform and target, respectively, $c(t)$ denotes clutter, and $n(t)$ represents noise. Suppose that $c(t)$ and $n(t)$ are Gaussian random processes with zero mean value, and the power spectral density is $S_{\mathrm{cc}}(f)$ and $S_{\mathrm{nn}}(f)$, respectively.

The transmitted waveform $x(t)$ and the interference signal $c(t)$ are convolved with the target $h(t)$ to obtain $z(t)$ and $d(t)$, respectively. After the addition of the above two with the noise $n(t)$, the echo $y(t)$ can be obtained after the ideal low-pass filter, and the duration of $y(t)$ is $T_{y}$, as shown in Figure 1.

Since the real radar target signal has a finite duration, $h(t)$ is a random process with finite energy, and it is assumed that any sample function of $h(t)$ can be integrated. The Fourier transform of the sample function $h(t)$ is $H(f)$. From Parseval's theorem,

$$
E_{h}=\int_{0}^{T_{h}}|h(t)|^{2} \mathrm{~d} t=\int_{-\infty}^{+\infty}|H(f)|^{2} \mathrm{~d} f .
$$

The energy spectral density of $h(t)$ is

$$
\xi_{H}(f)=E\left[|H(f)|^{2}\right] .
$$

The mean and variance are defined as

$$
\begin{aligned}
\mu_{H}(f) & =E[|H(f)|], \\
\sigma_{H}^{2}(f) & =E\left[\left|H(f)-\mu_{H}(f)\right|^{2}\right] .
\end{aligned}
$$

In this paper, it is assumed that $\mu_{H}(f)$ is 0 , so the energy spectral density (ESD) and the energy spectral variance (ESV) are equal. ESV describes the average energy of a random process with finite duration and zero mean value.

For the convolution of known signal $x(t)$ with random process $h(t)$, such as $z(t)=x(t) * h(t)$, the output ESV is

$$
\sigma_{Z}^{2}(f)=\sigma_{H}^{2}(f)|X(f)|^{2} .
$$

\section{Robust Waveform Design Based on MI}

3.1. Derivation of Mutual Information Formula. The mutual information to be researched in this paper is $I(h(t) ; y(t) \mid x(t))$ between target $h(t)$ and echo $y(t)$ when the transmitted waveform $x(t)$ is known.

Before solving mutual information, the basic knowledge of information theory needs to be introduced. Suppose $Y$ is a discrete random variable with a value range of $R_{Y}=\left\{y_{1}, y_{2}, y_{3}, \ldots \ldots\right\}$, and for each $y \in R_{Y}$, the probability of $Y=y$ is $P(y)$. The empirical and historical data

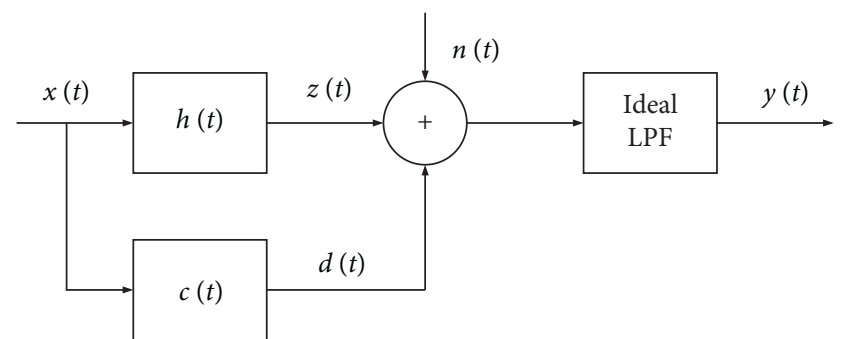

FIgURE 1: Signal model of random target with finite duration in clutter.

obtained before the experiment of obtaining samples are called prior information. In order to measure the size of the prior information, the self-information of $Y$ is defined as

$$
I(y)=-\log P(y) \text {. }
$$

In information theory, the logarithm base 2 is often used, and the unit is Bit. In this paper, for the convenience of calculation, the logarithm base $e$ is used, and the unit is Nat. Therefore, the discrete form and continuous form of information entropy $H(Y)$ are, respectively, as follows:

$$
\begin{aligned}
& H(Y)=-\sum_{Y \in R_{Y}} P(y) \ln P(y), \\
& H(Y)=-\int_{-\infty}^{+\infty} P(y) \ln P(y) \mathrm{d} y .
\end{aligned}
$$

The mutual information between $Y$ and $Z$ is

$$
I(Y ; Z)=H(Y)-H(Y \mid Z),
$$

where $Y$ is the sum of three Gaussian random variables with zero mean value, so it is also a Gaussian random variable with zero mean value. Considering that $Z$ and $D$ are statistically independent, and $N$ is also statistically independent, the variance of $Y$ is as follows:

$$
\sigma_{Y}^{2}=\sigma_{Z}^{2}+\sigma_{N}^{2}+\sigma_{D}^{2}
$$

where $Y$ is a Gaussian random variable, so $P(y)$ conforms to a normal distribution. Assume that the mean value is $\mu$ and the variance is $\sigma$, then

$$
\begin{array}{r}
P(y)=\frac{1}{\sqrt{2 \pi \sigma^{2}}} e^{-(y-\mu)^{2} / 2 \sigma^{2}}, \\
\\
\int_{-\infty}^{+\infty} P(y) \mathrm{d} y=1 .
\end{array}
$$

So,

$$
\begin{aligned}
H(Y) & =-\int_{-\infty}^{+\infty} P(y) \ln P(y) \mathrm{d} y=-\int_{-\infty}^{+\infty} P(y) \ln \frac{1}{\sqrt{2 \pi \sigma^{2}}} e^{-(y-\mu)^{2} / 2 \sigma^{2}} \mathrm{~d} y \\
& =-\int_{-\infty}^{+\infty} P(y)\left[\ln \frac{1}{\sqrt{2 \pi \sigma^{2}}}+\ln e^{-(y-\mu)^{2} / 2 \sigma^{2}}\right] \mathrm{d} y \\
& =\frac{1}{2} \ln \left(2 \pi \sigma^{2}\right)+\int_{-\infty}^{+\infty} \frac{(y-\mu)^{2}}{2 \sigma^{2}} \frac{1}{\sqrt{2 \pi \sigma^{2}}} e^{-(y-\mu)^{2} / 2 \sigma^{2}} \mathrm{~d} y .
\end{aligned}
$$


Create a function

$$
F(\mu, \sigma)=\int_{-\infty}^{+\infty} \frac{(y-\mu)^{2}}{2 \sigma^{2}} \frac{1}{\sqrt{2 \pi \sigma^{2}}} e^{-(y-\mu)^{2} / 2 \sigma^{2}} \mathrm{~d} y,
$$

with known

$$
\int_{-\infty}^{+\infty} e^{-y^{2}} \mathrm{~d} y=\sqrt{\pi}
$$

So,

$$
\begin{aligned}
\int_{-\infty}^{+\infty} e^{-y^{2}} y^{2} \mathrm{~d} y= & -\frac{1}{2} \int_{-\infty}^{+\infty} y d\left(e^{-y^{2}}\right) \mathrm{d} y \\
& =-\frac{1}{2}\left[y e^{-y^{2}}\right]_{-\infty}^{+\infty}-\frac{1}{2} \int_{-\infty}^{+\infty} e^{-y^{2}} \mathrm{~d} y=\frac{\sqrt{\pi}}{2}
\end{aligned}
$$

From the abovementioned equation, we can obtain

$$
\int_{-\infty}^{+\infty} e^{-y^{2}} y^{2} \mathrm{~d} y=\frac{\sqrt{\pi}}{2}
$$

Let us replace $y$ with $(y-\mu) / \sqrt{2} \sigma$; it can be calculated by

$$
\begin{gathered}
F(\mu, \sigma)=\frac{\sqrt{2} \sigma}{\sqrt{2 \pi \sigma^{2}}} \int_{-\infty}^{+\infty} \frac{(y-\mu)^{2}}{2 \sigma^{2}} e^{-(y-\mu)^{2} / 2 \sigma^{2}} \mathrm{~d}\left(\frac{y-\mu}{\sqrt{2} \sigma}\right) \\
=\frac{\sqrt{2} \sigma}{\sqrt{2 \pi \sigma^{2}}} \frac{\sqrt{\pi}}{2}=\frac{1}{2} .
\end{gathered}
$$

So,

$$
\begin{aligned}
H(Y)= & \frac{1}{2} \ln \left(2 \pi \sigma^{2}\right)+\int_{-\infty}^{+\infty} \frac{(y-\mu)^{2}}{2 \sigma^{2}} \frac{1}{\sqrt{2 \pi \sigma^{2}}} e^{-(y-\mu)^{2} / 2 \sigma^{2}} \mathrm{~d} y \\
& =\frac{1}{2} \ln \left(2 \pi \sigma^{2}\right)+\frac{1}{2}
\end{aligned}
$$

Because of the nature of information entropy, the constant can be ignored, so the information entropy can be obtained as

$$
H(Y)=\frac{1}{2} \ln \left(2 \pi \sigma^{2}\right)=\frac{1}{2} \ln \left[2 \pi\left(\sigma_{Z}^{2}+\sigma_{N}^{2}+\sigma_{D}^{2}\right)\right] .
$$

Similarly, the conditional entropy $H(Y \mid Z)$ between $Y$ and $Z$ is

$$
H(Y \mid Z)=\frac{1}{2} \ln \left[2 \pi\left(\sigma_{N}^{2}+\sigma_{D}^{2}\right)\right] .
$$

Mutual information $I(Y ; Z)$ can be obtained as

$$
I(Y ; Z)=H(Y)-H(Y \mid Z)=\frac{1}{2} \ln \left[1+\frac{\sigma_{Z}^{2}}{\sigma_{N}^{2}+\sigma_{D}^{2}}\right] \text {. }
$$

For the signals defined on the frequency interval $F_{k}=\left[f_{k}, f_{k}+\Delta f\right]$, such as $z_{k}(t), y_{k}(t), d_{k}(t)$, and $n_{k}(t)$, according to the sampling theorem, each signal can be replaced by a series of samples obtained from uniform sampling. Suppose that the sampling frequency of the signal is $2 \Delta f$. When $\Delta f$ is very small, the spectrum $X(f), Z(f)$,
$D(f), Y(f)$ is flat in $f \in F_{k}$ and can be approximated to a constant value. The Gaussian process samples sampled with uniform sampling rate $2 \Delta f$ are also statistically independent.

Sample $z_{k}(t)$ is an independent, identically distributed random variable, with zero mean value and variance $\sigma_{Z}^{2}$, and the total energy of $z_{k}(t)$ in the frequency interval $F_{k}$ is

$$
\varepsilon_{Z}\left(F_{k}\right)=\Delta f\left|X\left(f_{k}\right)\right|^{2} \sigma_{H}^{2}\left(f_{k}\right) .
$$

The number of sample points is $2 T_{y} \Delta f$, and the energy is uniformly distributed on independent sample points with the same distribution. So, the variance $\sigma_{Z}^{2}$ of each sample point is

$$
\sigma_{Z}^{2}=\frac{\varepsilon_{Z}\left(F_{k}\right)}{2 T_{y} \Delta f}=\frac{\Delta f\left|X\left(f_{k}\right)\right|^{2} \sigma_{H}^{2}\left(f_{k}\right)}{2 T_{y} \Delta f}=\frac{\left|X\left(f_{k}\right)\right|^{2} \sigma_{H}^{2}\left(f_{k}\right)}{2 T_{y}} .
$$
is

The total energy of the clutter process on time interval $T_{y}$

$$
\varepsilon_{D}\left(F_{k}\right)=\Delta f\left|X\left(f_{k}\right)\right|^{2} S_{\mathrm{cc}}\left(f_{k}\right) T_{y} .
$$

The variance of the clutter process at each sample point is

$$
\sigma_{D}^{2}=\frac{\varepsilon_{D}\left(F_{k}\right)}{2 T_{y} \Delta f}=\frac{\Delta f\left|X\left(f_{k}\right)\right|^{2} S_{\mathrm{cc}}\left(f_{k}\right) T_{y}}{2 T_{y} \Delta f}=\frac{\left|X\left(f_{k}\right)\right|^{2} S_{\mathrm{cc}}\left(f_{k}\right)}{2} \text {. }
$$

Similarly, the total energy of the noise process on time interval $T_{y}$ is

$$
\varepsilon_{N}\left(F_{k}\right)=S_{\mathrm{nn}}\left(f_{k}\right) T_{y} \Delta f
$$

Considering that the noise is Gaussian white noise, its power spectral density is a constant in the frequency domain, so

$$
\varepsilon_{N}\left(F_{k}\right)=S_{\mathrm{nn}}\left(f_{k}\right) T_{y} \Delta f=S_{\mathrm{nn}}(f) T_{y} \Delta f
$$

The variance of the noise process at each sample point is

$$
\sigma_{N}^{2}=\frac{\varepsilon_{N}\left(F_{k}\right)}{2 T_{y} \Delta f}=\frac{S_{\mathrm{nn}}(f) T_{y} \Delta f}{2 T_{y} \Delta f}=\frac{S_{\mathrm{nn}}(f)}{2} .
$$

Substituting $\sigma_{Z}^{2}, \sigma_{D}^{2}$, and $\sigma_{N}^{2}$ into formula (19), we get

$$
\begin{aligned}
I(Y ; Z) & =\frac{1}{2} \ln \left[1+\frac{\sigma_{Z}^{2}}{\sigma_{N}^{2}+\sigma_{D}^{2}}\right] \\
& =\frac{1}{2} \ln \left[1+\frac{\left(\left|X\left(f_{k}\right)\right|^{2} \sigma_{H}^{2}\left(f_{k}\right)\right) / 2 T_{y}}{\left(S_{\mathrm{nn}}(f) / 2\right)+\left(\left(\left|X\left(f_{k}\right)\right|^{2} S_{\mathrm{cc}}\left(f_{k}\right)\right) / 2\right)}\right],
\end{aligned}
$$

that is,

$$
I(Y ; Z)==\frac{1}{2} \ln \left[1+\frac{\left|X\left(f_{k}\right)\right|^{2} \sigma_{H}^{2}\left(f_{k}\right)}{T_{y}\left(S_{\mathrm{nn}}(f)+\left|X\left(f_{k}\right)\right|^{2} S_{\mathrm{cc}}\left(f_{k}\right)\right)}\right] .
$$


Within the observation interval $T_{y}$, there are statistically independent sample values with the number of $2 T_{y} \Delta f$, so the mutual information of $z_{k}(t)$ and $y_{k}(t)$ in the case of known $x_{k}(t)$ is

$$
I\left(y_{k}(t) ; z_{k}(t) \mid x_{k}(t)\right)=2 T_{y} \Delta f I(Y ; Z),
$$

that is,

$$
\begin{aligned}
& I\left(y_{k}(t) ; z_{k}(t) \mid x_{k}(t)\right) \\
& \quad=T_{y} \Delta f \ln \left[1+\frac{\left|X\left(f_{k}\right)\right|^{2} \sigma_{H}^{2}\left(f_{k}\right)}{T_{y}\left(S_{\mathrm{nn}}(f)+\left|X\left(f_{k}\right)\right|^{2} S_{\mathrm{cc}}\left(f_{k}\right)\right)}\right] .
\end{aligned}
$$

Within any frequency interval BW, it is divided into many disjoint intervals, and the interval bandwidth is $\Delta f$. When $\Delta f \longrightarrow 0$, the number of intervals is infinite, and the integral expression of mutual information can be obtained as

$$
\begin{aligned}
& I(y(t) ; z(t) \mid x(t)) \\
& \quad=T_{y} \int_{\mathrm{BW}} \ln \left[1+\frac{|X(f)|^{2} \sigma_{H}^{2}(f)}{T_{y}\left(S_{\mathrm{nn}}(f)+|X(f)|^{2} S_{\mathrm{cc}}(f)\right)}\right] \mathrm{d} f .
\end{aligned}
$$

3.2. Robust Water-Filling Waveform. In previous studies on waveform design, it is assumed that the prior information of the target spectrum is completely known. However, in actual scene, the real target spectrum cannot be captured with complete precision. It is assumed that the target spectrum exists in an uncertain range $\delta$, which is defined by the known upper and lower bounds, that is,

$$
H(f) \in \delta \Rightarrow\left\{l_{k} \leq H\left(f_{k}\right) \leq u_{k}, k=1,2,3 \ldots \ldots\right\},
$$

where $f_{k}$ is the sampling frequency, and for each sampling point, there is an upper bound and a lower bound. The confidence band of the target spectrum can be determined by spectrum estimation, so the upper and lower bounds of the estimated waveform spectrum are reasonable. In this paper, the upper and lower bounds are determined according to the uniform distribution function, which refers to the fact that the range of upper and lower bound is the random number consistent with uniform distribution within $[0,|H(f)|]$. So, the difference value of the spectrum corresponding to each sampling frequency may be different between the upper and lower bounds. The greater the difference between the upper and lower bounds of the uncertainty range is, the greater the uncertainty of the target spectrum is. For each specific target spectrum, there is an optimal transmitted waveform. However, the real target spectrum varies in the range of uncertainty, so in this paper, we adopt the maximin robust waveform design scheme.
For this, we first introduce the concept of robustness. Robustness is a term used in statistics to describe the insensitivity of control systems to perturbation of characteristics or parameters. In general, a robust signal processing scheme may not perform as well under nominal conditions as the optimal scheme under nominal conditions, but its overall performance will be good or acceptable relative to the defined feature categories. To achieve this, we must first specify a metric for the overall performance of a solution, which relates to a class of allowable conditions at the time of input. In many cases, a widely used and effective measure is the worst performance of a solution under certain input conditions. Obviously, if it performs well under most unfavorable condition, we can say that the given scheme is robust. Therefore, we propose the best performance scheme in the worst case, which is maximin robust scheme. The worst-case performance of this solution will be acceptable, which refers to the best performance that can be achieved under the most adverse conditions. So, this is not going to be very much lower than the optimal solution in the nominal case.

In the process of designing the robust transmitted waveform based on MI and SINR, the most unfavorable situation is the lower bound of the uncertainty range of the target waveform spectrum, that is, the lower bound of $H(f)$. Assume the real energy spectral density is $|H(f)|^{2}$, the upper bound is $\sigma_{\mathrm{U}}^{2}(f)$, and the lower bound is $\sigma_{\mathrm{L}}^{2}(f)$ :

$$
\begin{aligned}
& \left.\operatorname{MI}\left(\left|X^{\max \min }(f)\right|^{2}, \sigma_{\mathrm{U}}^{2}(f)\right)\left|\int_{\mathrm{BW}}\right| X^{\max \min }(f)\right|^{2} \mathrm{~d} f \leq E_{x} \\
& \geq\left.\operatorname{MI}\left(\left|X^{\max \min }(f)\right|^{2}, \sigma_{\mathrm{H}}^{2}(f)\right)\right|_{\int_{\mathrm{BW}}\left|X^{\max m i n}(f)\right|^{2} \mathrm{~d} f \leq E_{x}} \\
& \geq\left.\operatorname{MI}\left(\left|X^{\max \min }(f)\right|^{2}, \sigma_{\mathrm{L}}^{2}(f)\right)\right|_{\int_{\mathrm{BW}}\left|X^{\max \min }(f)\right|^{2} \mathrm{~d} f \leq E_{x}} \\
& \geq\left.\operatorname{MI}\left(|X(f)|^{2}, \sigma_{\mathrm{L}}^{2}(f)\right)\right|_{\int_{\mathrm{BW}}|X(f)|^{2} \mathrm{~d} f \leq E_{x} .}
\end{aligned}
$$

To solve the formula,

$$
\max \left\{\left.\min _{H(f) \in \delta} \operatorname{MI}\left(|X(f)|^{2}, \sigma_{\mathrm{L}}^{2}(f)\right)\left|\int_{\mathrm{BW}}\right| X(f)\right|^{2} \mathrm{~d} f \leq E_{x}\right\},
$$

that is,

$$
\begin{array}{ll}
\max & T_{y} \int_{\mathrm{BW}} \ln \left[1+\frac{|X(f)|^{2} \sigma_{\mathrm{L}}^{2}(f)}{T_{y}\left(S_{\mathrm{nn}}(f)+|X(f)|^{2} S_{\mathrm{cc}}(f)\right)}\right] \mathrm{d} f \\
\text { s.t. } \quad \int_{\mathrm{BW}}|X(f)|^{2} \mathrm{~d} f \leq E_{x}
\end{array}
$$


The following function is established by using the Lagrange multiplier method:

$$
L\left(|X(f)|^{2}, \lambda\right)=T_{y} \int_{\mathrm{BW}} \ln \left[1+\frac{|X(f)|^{2} \sigma_{\mathrm{L}}^{2}(f)}{T_{y}\left(S_{\mathrm{nn}}(f)+|X(f)|^{2} S_{\mathrm{cc}}(f)\right)}\right] \mathrm{d} f-\lambda\left(\int_{\mathrm{BW}}|X(f)|^{2} \mathrm{~d} f-E_{x}\right) .
$$

After removing the integral sign and constant, it is equivalent to maximizing the function $l\left(|X(f)|^{2}\right)$ with the energy spectrum $|X(f)|^{2}$ of transmitted waveform, which can be expressed by the following equation:

$$
l\left(|X(f)|^{2}\right)=T_{y} \ln \left[1+\frac{|X(f)|^{2} \sigma_{\mathrm{L}}^{2}(f)}{T_{y}\left(S_{\mathrm{nn}}(f)+|X(f)|^{2} S_{\mathrm{cc}}(f)\right)}\right]-\lambda|X(f)|^{2} .
$$

The above-given formula is too complicated, so we adopt the method of symbol substitution to simplify the formula. Let $|X(f)|^{2}=x, \quad \sigma_{\mathrm{L}}^{2}(f)=h, S_{\mathrm{cc}}(f)=c, S_{\mathrm{nn}}(f)=n$, and $T_{y}=t$; then, equation (37) becomes

$$
l(x)=t \ln \left[1+\frac{h x}{t(c x+n)}\right]-\lambda x
$$

Derive $l(x)$ to $x$ as follows:

$$
\frac{\mathrm{d}(l(x))}{\mathrm{d} x}=t \frac{1}{1+(h x / t(c x+n))} \frac{h t(c x+n)-h t c x}{t^{2}(c x+n)^{2}}-\lambda .
$$

Let $A=T_{y} / \lambda$, and set the derivative function to zero to find stagnation point, so

$$
\frac{h n t}{[h x+t(n+c x)](c x+n)}-\frac{t}{A}=0
$$

that is,

$$
\left(h c+t c^{2}\right) x^{2}+(h n+2 t c n) x+t n^{2}-h n A=0 .
$$

Then,

$$
x=\frac{-(h n+2 t c n) \pm \sqrt{(h n+2 t c n)^{2}-4\left(h c+t c^{2}\right)\left(t n^{2}-h n A\right)}}{2\left(h c+t c^{2}\right)} .
$$

Leaving out the minus sign, we can get

$$
x=-\frac{n(h+2 t c)}{2 c(h+t c)}+\sqrt{\frac{h^{2} n^{2}+4 A n c h^{2}+4 A h n t c^{2}}{4\left(h c+t c^{2}\right)^{2}}} .
$$

Let

$$
\begin{aligned}
& d=\frac{t n}{h} \\
& r=\frac{n(h+2 t c)}{2 c(h+t c)} \\
& s=\frac{n h}{c(h+t c)} .
\end{aligned}
$$

Then,

$$
x=-r+\sqrt{r^{2}+s(A-d)}
$$

Since the power spectrum density of the transmitted signal is nonnegative, the robust waveform can be expressed as

$$
\left|X^{\max \min }(f)\right|^{2}=\max \left[-R(f)+\sqrt{R^{2}(f)+S(f)(A-D(f))}, 0\right],
$$

where

$$
\begin{aligned}
& D(f)=\frac{T_{y} S_{\mathrm{nn}}(f)}{\sigma_{\mathrm{L}}^{2}(f)}, \\
& R(f)=\frac{S_{\mathrm{nn}}(f)\left(\sigma_{\mathrm{L}}^{2}(f)+2 T_{y} S_{\mathrm{cc}}(f)\right)}{2 S_{\mathrm{cc}}(f)\left(\sigma_{\mathrm{L}}^{2}(f)+T_{y} S_{\mathrm{cc}}(f)\right)}, \\
& S(f)=\frac{S_{\mathrm{nn}}(f) \sigma_{\mathrm{L}}^{2}(f)}{S_{\mathrm{cc}}(f)\left(\sigma_{\mathrm{L}}^{2}(f)+T_{y} S_{\mathrm{cc}}(f)\right)}
\end{aligned}
$$

$A$ can be obtained from the following energy constraints:

$$
\text { s.t. } \int_{\mathrm{BW}} \max \left[-R(f)+\sqrt{R^{2}(f)+S(f)(A-D(f))}, 0\right] \mathrm{d} f \leq E_{x} \text {. }
$$

\subsection{First-Order Taylor Approximation. Assume that}

$$
\left|X^{\max \min }(f)\right|^{2}=-R(f)+\sqrt{R^{2}(f)+S(f)(A-D(f))} .
$$

Taylor's first-order approximation is the first two terms of Taylor expansion:

$$
f(x)=f^{\prime}\left(x_{0}\right) x+f\left(x_{0}\right) .
$$

Let $A-D(f)=x, R(f)=r$, and $S(f)=s$; then,

$$
\left|X^{\max \min }(f)\right|^{2}=-r+\sqrt{r^{2}+s(A-d)} \text {. }
$$

Derive $\left|X^{\max \min }(f)\right|^{2}$ to $x$ as follows:

$$
\frac{\mathrm{d}\left|X^{\max \min }(f)\right|^{2}}{\mathrm{~d} x}=\frac{s}{2 \sqrt{r^{2}+s x}} .
$$

Taylor's expansion at $x_{0}=0$ is

$$
\left.\frac{\mathrm{d}\left|X^{\max \min }(f)\right|^{2}}{\mathrm{~d} x}\right|_{x=0}=\frac{s}{2 r}=\frac{n h /(c(h+t c))}{(n(h+2 t c)) /(c(h+t c))}=\frac{h}{h+2 t c} .
$$

Let

$$
b=\frac{h}{h+2 t c} .
$$


Then,

$$
\left|X^{\max \min }(f)\right|^{2}=b x
$$

that is,

$$
\left|X^{\max \min }(f)\right|^{2}=B(f)(A-D(f))
$$

where

$$
B(f)=\frac{\sigma_{\mathrm{L}}^{2}(f)}{\sigma_{\mathrm{L}}^{2}(f)+2 T_{y} S_{\mathrm{cc}}(f)}
$$

\section{Robust Waveform Design Based on SINR}

It can be seen from the signal model in Figure 1 that

$$
y(t)=z(t)+d(t)+n(t) .
$$

The definition of SINR is the ratio of the useful signal power in the echo to the power of the interference signal plus the noise signal:

$$
\operatorname{SINR}=\int_{\mathrm{BW}} \frac{\sigma_{Z}^{2}(f)}{S_{\mathrm{nn}}(f)+\sigma_{D}^{2}(f)} \mathrm{d} f,
$$

where

$$
\begin{aligned}
\sigma_{Z}^{2}(f) & =|X(f)|^{2} \sigma_{\mathrm{H}}^{2}(f), \\
\sigma_{D}^{2}(f) & =|X(f)|^{2} S_{\mathrm{cc}}(f) .
\end{aligned}
$$

So,

$$
\operatorname{SINR}=\int_{\mathrm{BW}} \frac{|X(f)|^{2} \sigma_{\mathrm{H}}^{2}(f)}{S_{\mathrm{nn}}(f)+|X(f)|^{2} S_{\mathrm{cc}}(f)} \mathrm{d} f .
$$

Similarly, from the above derivation of MI robust waveform, it can be seen that, for SINR, the spectrum corresponding to each sampling frequency still has upper and lower bounds:

$$
\begin{aligned}
& \left.\operatorname{SINR}\left(\left|X^{\max \min }(f)\right|^{2}, \sigma_{\mathrm{U}}^{2}(f)\right)\right|_{\int_{\mathrm{BW}}\left|X^{\max \min }(f)\right|^{2} \mathrm{~d} f \leq E_{x}} \\
& \geq\left.\operatorname{SINR}\left(\left|X^{\max \min }(f)\right|^{2}, \sigma_{\mathrm{H}}^{2}(f)\right)\right|_{\int_{\mathrm{BW}}\left|X^{\max \min }(f)\right|^{2} \mathrm{~d} f \leq E_{x}} \\
& \geq\left.\operatorname{SINR}\left(\left|X^{\max \min }(f)\right|^{2}, \sigma_{\mathrm{L}}^{2}(f)\right)\right|_{\int_{\mathrm{BW}}\left|X^{\max \min }(f)\right|^{2} \mathrm{~d} f \leq E_{x}} \\
& \geq\left.\operatorname{SINR}\left(|X(f)|^{2}, \sigma_{\mathrm{L}}^{2}(f)\right)\right|_{\int_{\mathrm{BW}}|X(f)|^{2} \mathrm{~d} f \leq E_{x} \cdot}
\end{aligned}
$$

The problem of optimizing the SINR can be expressed as

$$
\begin{array}{ll}
\max & \int_{\mathrm{BW}} \frac{|X(f)|^{2} \sigma_{\mathrm{L}}^{2}(f)}{(f)+|X(f)|^{2} S_{\mathrm{cc}}(f)} \mathrm{d} f \\
\text { s.t. } & \int_{\mathrm{BW}}|X(f)|^{2} \mathrm{~d} f \leq E_{x}
\end{array}
$$

The following function is established by using the Lagrange multiplier method:

$$
\begin{aligned}
L\left(|X(f)|^{2}, \lambda\right)= & \int_{\mathrm{BW}} \frac{|X(f)|^{2} \sigma_{\mathrm{Ln}}^{2}(f)+|X(f)|^{2} S_{\mathrm{cc}}(f)}{d f} \\
& -\lambda\left(\int_{\mathrm{BW}}|X(f)|^{2} \mathrm{~d} f-E_{x}\right) .
\end{aligned}
$$

After removing the integral sign and constant, it is equivalent to maximizing the function $l\left(|X(f)|^{2}\right)$ with the energy spectrum $|X(f)|^{2}$ of the transmitted waveform, which can be expressed by the following equation:

$$
l\left(|X(f)|^{2}\right)=\frac{|X(f)|^{2} \sigma_{\mathrm{L}}^{2}(f)}{S_{\mathrm{nn}}(f)+|X(f)|^{2} S_{\mathrm{cc}}(f)}-\lambda|X(f)|^{2} .
$$

The above formula is too complicated, so we take the method of symbol substitution to simplify the formula. Let $|X(f)|^{2}=x, \sigma_{\mathrm{L}}^{2}(f)=h, S_{\mathrm{cc}}(f)=c, S_{\mathrm{nn}}(f)=n$, and $T_{y}=t$; then, the above equation becomes

$$
l(x)=\frac{h x}{c x+n}-\lambda x
$$

Derive $l(x)$ to $x$ as follows:

$$
\frac{\mathrm{d}(l(x))}{\mathrm{d} x}=\frac{h(c x+n)-h c x}{(c x+n)^{2}}-\lambda .
$$

We let $A=\sqrt{1 / \lambda}$ and set the derivative function to zero to find stagnation point, so

$$
\frac{n h}{(c x+n)^{2}}=\frac{1}{A^{2}}
$$

that is,

$$
c^{2} x^{2}+2 c n x+n^{2}-n h A^{2}=0 .
$$

Then,

$$
x=\frac{-2 c n \pm \sqrt{4 c^{2} n^{2}-4 c^{2}\left(n^{2}-n h A^{2}\right)}}{2 c^{2}} .
$$

Leaving out the minus sign, we get

$$
x=-\frac{n}{c}+\sqrt{\frac{4 n h A^{2} c^{2}}{4 c^{4}}}=\frac{A \sqrt{n h}-n}{c} .
$$

Set

$$
\begin{aligned}
& d=\sqrt{\frac{n}{h}}, \\
& b=\frac{\sqrt{n h}}{c} .
\end{aligned}
$$

Then,

$$
x=b(A-d) .
$$

Since the power spectrum density of the transmitted signal is nonnegative, the robust waveform can be expressed as

$$
\left|X^{\max \min }(f)\right|^{2}=\max [B(f)(A-D(f)), 0],
$$

where 


$$
\begin{aligned}
& D(f)=\sqrt{\frac{S_{\mathrm{nn}}(f)}{\sigma_{\mathrm{L}}^{2}(f)},} \\
& B(f)=\frac{\sqrt{S_{\mathrm{nn}}(f) \sigma_{\mathrm{L}}^{2}(f)}}{S_{\mathrm{cc}}(f)} .
\end{aligned}
$$

$A$ can be obtained from the following energy constraints:

$$
\text { s.t. } \int_{\mathrm{BW}} \max [B(f)(A-D(f)), 0] \mathrm{d} f \leq E_{x} \text {. }
$$

\section{Search of Lagrange Multipliers Based on Bisection Algorithm}

The abovementioned robust transmitted waveform based on SINR and MI has been obtained as

$$
\left|X^{\max \min }(f)\right|^{2}=\max [B(f)(A-D(f)), 0],
$$

where $B(f), A$, and $D(f)$ have different values based on different criteria, and $A=1 / \lambda$ based on SINR, $A=T_{y} / \lambda$ based on MI. As for how to find the value of $A$, this paper adopts successive bisection algorithm to search it.

The idea of bisection algorithm is continuous split in half, which is a very classic algorithm. We suppose that $[a, b]$ is the closed interval over the real number field, and define the interval sequence as follows: $\left[a_{n}, b_{n}\right], a_{0}=a$, and $b_{0}=b$. And for any natural number $n,\left[a_{n+1}, b_{n+1}\right]$ is equal to $\left[a_{n}, c_{n}\right]$ or $\left[c_{n}, b_{n}\right]$, where $c_{n}$ is the midpoint of $\left[a_{n}, b_{n}\right]$. When using bisection algorithm to search approximation, the data should be arranged in order of size.

Examples are given to illustrate the realization process of bisection algorithm, such as using bisection algorithm to find the zero point of the function. Set the function $f(x)=x^{4}+x-1$. Because the function is continuous, and $f(0)<0, f(1)>0$, the function must have zero point in the interval $[0,1]$. Now, use bisection algorithm to find the zero point, take the midpoint 0.5 of interval $[0,1]$, and get $f(0.5)<0$. Therefore, we can narrow down the range of interval. Next, take the midpoint 0.75 of interval $[0.5,1]$, and continue to compare $f(0.75)$ with 0 , until the error is within the allowable range; then, we can consider this point as the zero point of the function. Figure 2 shows a flowchart of bisection algorithm.

The principle used to search Lagrange multiplier $A$ is similar. Find the value interval of $A$, take the midpoint, substitute in to find $\left|X^{\max \min }(f)\right|^{2}$, and compare the relationship between $\int_{\mathrm{BW}}\left|X^{\max \min }(f)\right|^{2} \mathrm{~d} f$ and $E_{x}$. Make successive approximation by bisection algorithm and assume the allowable error is eps, until $\left.\left|\int_{\mathrm{BW}}\right| X^{\max \min }(f)\right|^{2} \mathrm{~d} f-E_{x} \mid \leq$ eps, and it can be considered that $A$ meets the condition.

In this paper, the scope of $A$ is shown as follows:

$$
\begin{aligned}
& A_{\min }=\min [D(f)], \\
& A_{\max }=\frac{E_{x}}{\mathrm{BW} \min [B(f)]}+\max [D(f)] .
\end{aligned}
$$

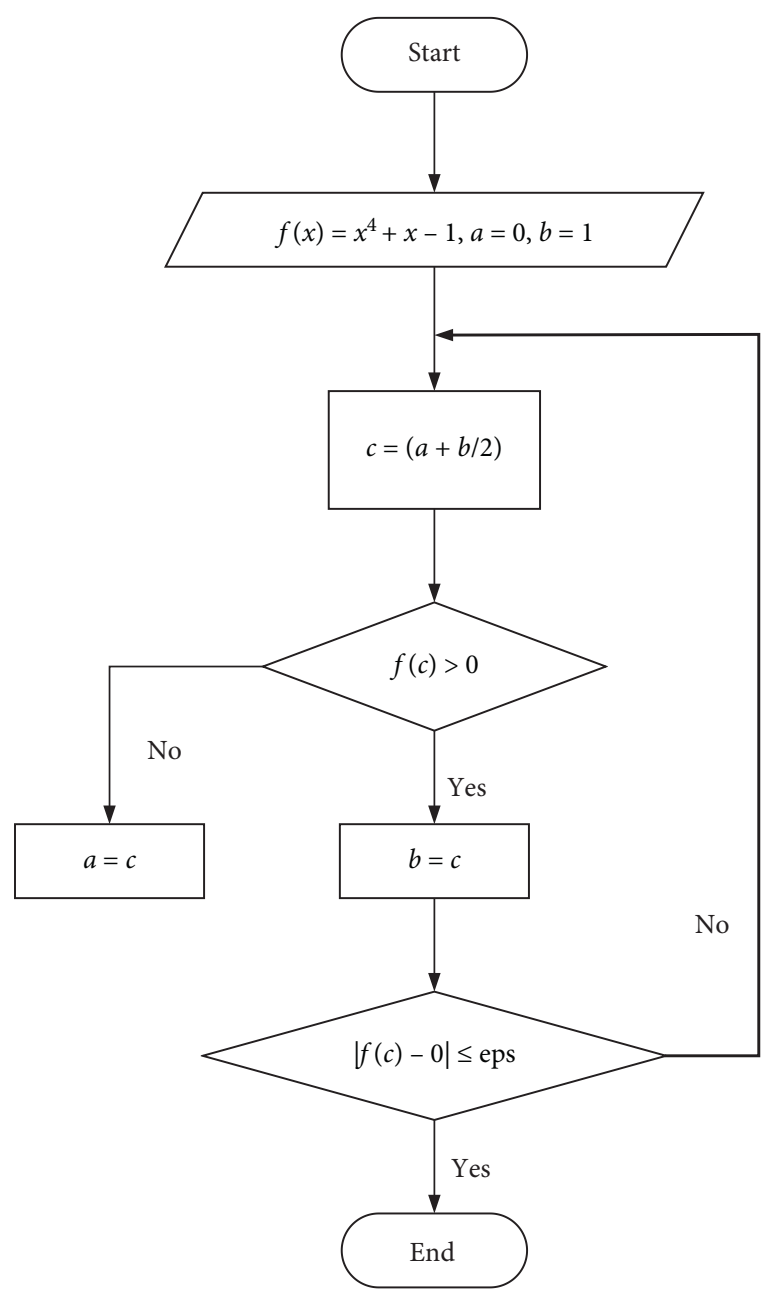

Figure 2: Flowchart of bisection algorithm.

Let $a_{0}=A_{\min }$ and $b_{0}=A_{\max }$. By using the energy constraint, the value of the optimal $A$ can be approached step by step.

\section{Maximum Marginal Allocation}

The amount of a certain resource is limited. If resources are invested in a variety of activities, the problem of how to allocate resources to achieve the optimal total effect will arise. This is resource allocation problem. We can regard the energy allocation of transmitted waveform as a resource allocation problem, which solves the optimization problem of a single constraint. That is, how to allocate the energy of transmitted waveform to achieve the most effective result under the condition of energy constraint.

As for how to allocate the energy of the transmitted waveform in a certain frequency band, we can regard it as a multistage decision process of dynamic programming. In each stage, a decision needs to be made on the allocation of energy. The finite energy $E_{x}$ is allocated to different $f_{k}$ to maximize the overall mutual information or signal-to-interference-plus-noise ratio. obtain

The integral form of mutual information is discretized to 


$$
\mathrm{MI}=T_{y} \sum_{k=1}^{N} \ln \left[1+\frac{\left|X\left(f_{k}\right)\right|^{2} \sigma_{\mathrm{L}}^{2}\left(f_{k}\right)}{T_{y}\left(S_{\mathrm{nn}}\left(f_{k}\right)+\left|X\left(f_{k}\right)\right|^{2} S_{\mathrm{cc}}\left(f_{k}\right)\right)}\right] \Delta f \text {. }
$$

Similarly,

$$
\operatorname{SINR}=\sum_{k=1}^{N} \frac{\left|X\left(f_{k}\right)\right|^{2} \sigma_{\mathrm{L}}^{2}\left(f_{k}\right)}{S_{\mathrm{nn}}\left(f_{k}\right)+\left|X\left(f_{k}\right)\right|^{2} S_{\mathrm{cc}}\left(f_{k}\right)} \Delta f,
$$

where

$$
\begin{aligned}
& f_{k}=k \Delta f, \\
& \Delta f=\frac{B W}{N} .
\end{aligned}
$$

The above formula is too complex, and we adopt the method of symbol substitution to simplify the formula. Let

$$
\begin{aligned}
& x(k)=\left|X\left(f_{k}\right)\right|^{2}, \\
& \alpha_{1}(k)=\frac{T_{y} S_{\mathrm{cc}}\left(f_{k}\right)}{\sigma_{\mathrm{L}}^{2}\left(f_{k}\right)}, \\
& \alpha_{2}(k)=\frac{S_{\mathrm{cc}}\left(f_{k}\right)}{\sigma_{\mathrm{L}}^{2}\left(f_{k}\right)}, \\
& \beta_{1}(k)=\frac{T_{y} S_{\mathrm{nn}}\left(f_{k}\right)}{\sigma_{\mathrm{L}}^{2}\left(f_{k}\right)}, \\
& \beta_{2}(k)=\frac{S_{\mathrm{nn}}\left(f_{k}\right)}{\sigma_{\mathrm{L}}^{2}\left(f_{k}\right)} .
\end{aligned}
$$

So, MI and SINR can be abbreviated as

$$
\begin{aligned}
\mathrm{MI} & =T_{y} \sum_{k=1}^{N} \ln \left[1+\frac{x(k)}{\alpha_{1}(k) x(k)+\beta_{1}(k)}\right] \Delta f, \\
\mathrm{SINR} & =\sum_{k=1}^{N} \frac{x(k)}{\alpha_{2}(k) x(k)+\beta_{2}(k)} \Delta f .
\end{aligned}
$$

Energy constraint is

$$
\sum_{k=1}^{N} x(k)=\frac{E_{x}}{\Delta f}=X_{\max }
$$

Under the above energy constraints, we seek the maximization of

$$
\sum_{k=1}^{N} f(x(k), k)
$$

where

$$
\begin{aligned}
f_{\mathrm{MI}}(x(k), k) & =\ln \left[1+\frac{x(k)}{\alpha_{1}(k) x(k)+\beta_{1}(k)}\right], \\
f_{\mathrm{SINR}}(x(k), k) & =\frac{x(k)}{\alpha_{2}(k) x(k)+\beta_{2}(k)} .
\end{aligned}
$$

The minimum energy distribution unit is defined as $\varepsilon_{0}$, and the number of energy components is defined as $P$.

So,

$$
\begin{array}{r}
X_{\max }=P \varepsilon_{0}, \\
0 \leq x(k) \leq X_{\max },
\end{array}
$$

$x(k)$ can be selected in set $\left\{0, \varepsilon_{0}, 2 \varepsilon_{0}, 3 \varepsilon_{0}, \ldots \ldots, P \varepsilon_{0}\right\}$. The core idea of MMA algorithm is to allocate the energy of $\varepsilon_{0}$ in each step and allocate all the energy after step $P$. When $\varepsilon_{0}$ is set to be very small, $P$ is very large, and the energy allocated in each step will have a tiny impact. We choose the serial number $k$ corresponding to the impact that maximizes mutual information to allocate, so that each step is optimal, so as to achieve the purpose of the overall optimal.

In the first step, for any $k \neq j$, if $f\left(\varepsilon_{0}, j\right)>f\left(\varepsilon_{0}, k\right)$, it is optimal for $k=j$. Let $x(j)=\varepsilon_{0}$, and assign a unit of energy to it. Since the first step has already allocated a share of the energy, in the second step, for $k=j$, we compare the edge increase by $f\left(2 \varepsilon_{0}, j\right)-f\left(\varepsilon_{0}, j\right)$. So, $\left\{f\left(\varepsilon_{0}, 1\right), f\left(\varepsilon_{0}, 2\right), \ldots \ldots, f\left(\varepsilon_{0}, j-\right.\right.$ $\left.1), f\left(2 \varepsilon_{0}, j\right)-f\left(\varepsilon_{0}, j\right), f\left(\varepsilon_{0}, j+1\right), \ldots \ldots, f\left(\varepsilon_{0}, N\right)\right\}$. The second step is to find the maximum value in the above set and assign a unit of energy to it. Similarly, after step $P$, all the energy is allocated, that is,

$$
\begin{array}{r}
x(k)=a_{k} \varepsilon_{0},(k=1,2, \ldots, N), \\
\sum_{k=1}^{N} a_{k}=P .
\end{array}
$$

The flowchart is shown in Figure 3.

In order to understand the algorithm of MMA, an example is given. For example, the frequency domain is discretized into four components $(N=4)$. Let $X_{\max }=5$, which is $x(1)+x(2)+x(3)+x(4)=5$. To maximize $M$,

$$
M=f(x(1), 1)+f(x(2), 2)+f(x(3), 3)+f(x(4), 4)
$$

According to the different criteria MI and SINR, $M$ can be divided into

$$
\begin{array}{r}
M_{1}=\sum_{k=1}^{4} \ln \left[1+\frac{x(k)}{\alpha_{1}(k) x(k)+\beta_{1}(k)}\right], \\
M_{2}=\sum_{k=1}^{4} \frac{x(k)}{\alpha_{2}(k) x(k)+\beta_{2}(k)} .
\end{array}
$$

Let $\varepsilon_{0}=1$, so with total energy $X_{\max }=5$, you can distribute $0,1,2,3,4$, or 5 units of energy to $x(1), x(2), x(3)$, or $x(4)$.

The corresponding values of $f_{\mathrm{MI}}(x(k), k)$ and $f_{\text {SINR }}(x(k), k)$ are shown in Tables 1 and 2, when initial allocation of energy. The underlined value is the maximum marginal growth at this time.

Taking mutual information as an example, the realization process of MMA algorithm is illustrated. When the initial energy is allocated by Table 1 , for $k=1,2,3,4$, $f_{\mathrm{MI}}(x(k), k)$ is $0.0708,0.0793,0.0880,0.0969$, respectively. Find the maximum value 0.0969 and allocate a unit of energy 


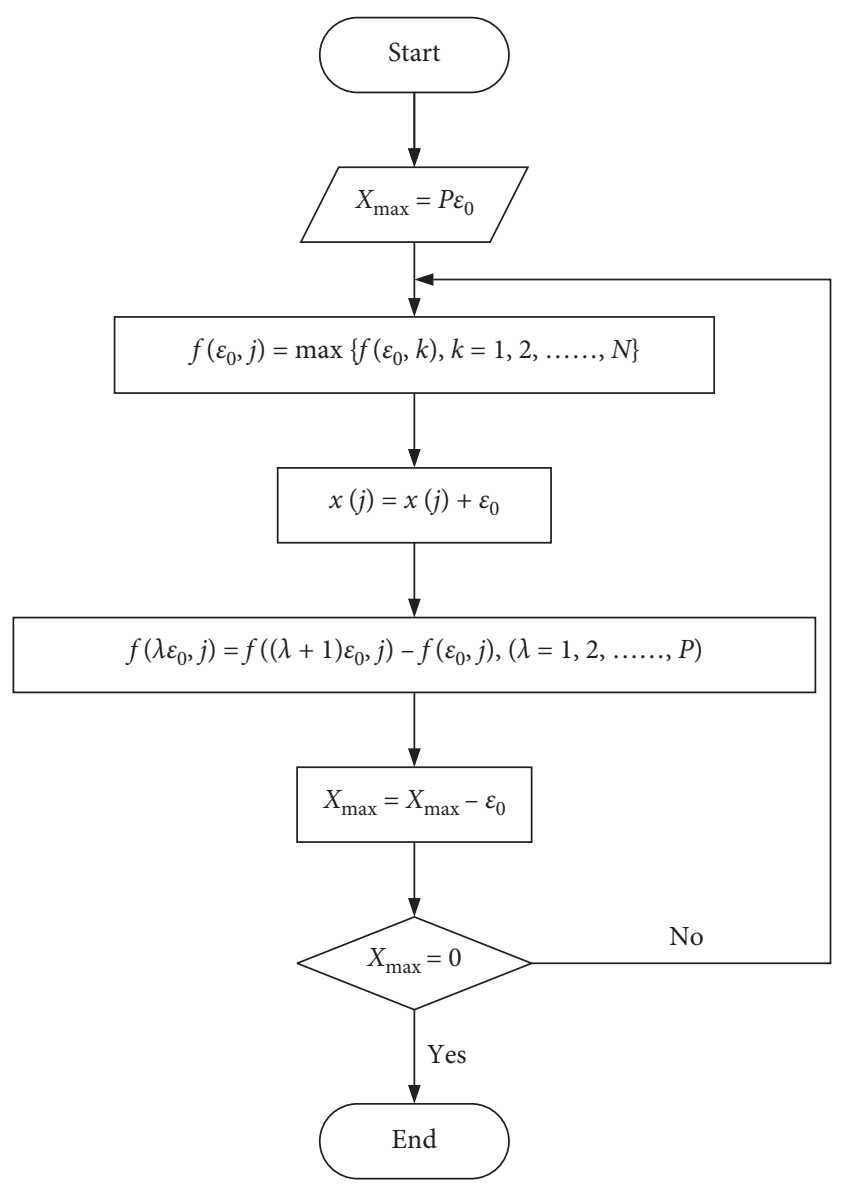

FIgURE 3: Flowchart of MMA algorithm.

TABLE 1: $f_{\text {MI }}(x(k), k)$ corresponding to different $x(k)$.

\begin{tabular}{ccccc}
\hline & $k=1$ & $k=2$ & $k=3$ & $k=4$ \\
\hline$x(k)=0$ & 0 & 0 & 0 & 0 \\
$x(k)=1$ & 0.0708 & 0.0793 & 0.0880 & 0.0969 \\
$x(k)=2$ & 0.0892 & 0.0998 & 0.1108 & 0.1219 \\
$x(k)=3$ & 0.0977 & 0.1092 & 0.1212 & 0.1334 \\
$x(k)=4$ & 0.1025 & 0.1147 & 0.1272 & 0.1400 \\
$x(k)=5$ & 0.1057 & 0.1182 & 0.1311 & 0.1443 \\
\hline
\end{tabular}

TABLE 2: $f_{\text {SINR }}(x(k), k)$ corresponding to different $x(k)$.

\begin{tabular}{ccccc}
\hline & $k=1$ & $k=2$ & $k=3$ & $k=4$ \\
\hline$x(k)=0$ & 0 & 0 & 0 & 0 \\
$x(k)=1$ & 0.7337 & 0.8248 & 0.9200 & 1.0177 \\
$x(k)=2$ & 0.9329 & 1.0495 & 1.1714 & 1.2966 \\
$x(k)=3$ & 1.0258 & 1.1543 & 1.2888 & 1.4270 \\
$x(k)=4$ & 1.0795 & 1.2150 & 1.3568 & 1.5026 \\
$x(k)=5$ & 1.1146 & 1.2546 & 1.4012 & 1.5519 \\
\hline
\end{tabular}

to $k=4$. When the second energy is allocated by Table 3 , the key is the marginal growth when $k=4$. Find the maximum value 0.0880 and allocate a unit of energy to $k=3$. Similarly, the next steps are the same. Tables 4-6 summarize the final energy distribution. In the end, the maximum mutual information is 0.3600 and two parts of energy are allocated to $k=4$, and one part of energy is allocated to all the other parts, which is

$$
x(1)=x(2)=x(3)=\varepsilon_{0}, x(4)=2 \varepsilon_{0} .
$$

\section{Simulation Results and Analysis}

7.1. SINR and MI in Three Cases. The power spectral density of noise is

$$
S_{\mathrm{nn}}(f)=1 \text {. }
$$

The frequency range is $[-0.5,0.5]$, and the number of sampling points is $N=256$. The energy range of the transmitted waveform is $[1,10]$. It is assumed that the upper and lower bounds of the uncertainty range of the target spectrum conform to the uniform distribution on $[0,|H(f)|]$.

As can be seen from Figure 4, the target spectrum is mainly concentrated around the frequency of -0.2 and 0.4 and is less subject to clutter interference around 0.2. Therefore, the energy distribution of the transmitted waveform is mainly concentrated around $-0.2,0.2$, and 0.4 , so that a larger mutual information or signal-to-interference-plus-noise ratio can be obtained, so as to obtain better target estimation performance.

Under the three conditions including known target spectrum, known target spectrum lower bound (worst case), and known target spectrum upper bound (best case), and energy from 1 to 10 is allocated to the transmitted waveform, we compare the size of SINR.

From the above Figures 5-7, we can conclude that the SINR obtained from the upper bound waveform and the optimal waveform is very close when the target spectrum is known or under the most favorable case (upper bound). When the target spectrum is known, the SINR obtained from the optimal waveform is larger, and under the most favorable case (upper bound), the SINR obtained from the upper bound waveform is larger. However, in real scenarios, the real target spectrum cannot be captured with complete precision. In the worst case (lower bound), the SINR is the largest by using the robust waveform, and the difference is more significant than the other two waveforms.

Similarly, the comparison on MI is similar under three conditions including the known target spectrum, the known target spectrum lower bound (the worst case), and the known target spectrum upper bound (the best case).

From Figures 8-10, we can still conclude that when the target spectrum is known or under the most favorable case (upper bound), the MI obtained by the upper bound waveform is very close to that obtained by the optimal waveform. However, in the worst case (lower bound), the maximum MI is obtained by using the robust waveform, and the difference is more significant than by the other two waveforms. Therefore, the robust waveform design scheme based on SINR and MI can improve the performance of radar system under the most unfavorable condition. 
TABLE 3: The marginal value of $f_{\mathrm{MI}}(x(k), k)$ after the first energy allocation.

\begin{tabular}{lcccc}
\hline & $k=1$ & $k=2$ & $k=3$ & $k=4$ \\
\hline$x(k)=0$ & 0 & 0 & 0 & 0 \\
$x(k)=1$ & 0.0708 & 0.0793 & 0.0880 & 0.1108 \\
$x(k)=2$ & 0.0892 & 0.0998 & 0.1212 & 0.0365 \\
$x(k)=3$ & 0.0977 & 0.1092 & 0.1272 & 0.0431 \\
$x(k)=4$ & 0.1025 & 0.1147 & 0.1311 & 0.0474 \\
$x(k)=5$ & 0.1057 & 0.1182 & - \\
\hline
\end{tabular}

TABLE 4: The marginal value of $f_{\mathrm{MI}}(x(k), k)$ after the second energy allocation.

\begin{tabular}{lcccc}
\hline & $k=1$ & $k=2$ & $k=3$ & $k=4$ \\
\hline$x(k)=0$ & 0 & 0 & 0 & 0 \\
$x(k)=1$ & 0.0708 & 0.0793 & 0.0228 & 0.0332 \\
$x(k)=2$ & 0.0892 & 0.0998 & 0.0392 & 0.0365 \\
$x(k)=3$ & 0.0977 & 0.1092 & 0.0431 & 0.0431 \\
$x(k)=4$ & 0.1025 & 0.1147 & - & 0.0474 \\
$x(k)=5$ & 0.1057 & 0.1182 & & - \\
\hline
\end{tabular}

TABLE 5: The marginal value of $f_{\mathrm{MI}}(x(k), k)$ after the third energy allocation.

\begin{tabular}{lcccc}
\hline & $k=1$ & $k=2$ & $k=3$ & $k=4$ \\
\hline$x(k)=0$ & 0 & 0 & 0 & 0 \\
$x(k)=1$ & 0.0708 & 0.0205 & 0.0228 & 0.0332 \\
$x(k)=2$ & 0.0892 & 0.0299 & 0.0392 & 0.0365 \\
$x(k)=3$ & 0.0977 & 0.0354 & 0.0431 & 0.0431 \\
$x(k)=4$ & 0.1025 & 0.0389 & - & 0.0474 \\
$x(k)=5$ & 0.1057 & - & - \\
\hline
\end{tabular}

TABLE 6: Final energy allocation.

\begin{tabular}{|c|c|c|c|c|c|}
\hline Step & $k=1$ & $k=2$ & $k=3$ & $k=4$ & $f_{M I}$ \\
\hline 1 & & & & $\varepsilon_{0}$ & 0.0969 \\
\hline 2 & & & $\varepsilon_{0}$ & & 0.1849 \\
\hline 3 & & $\varepsilon_{0}$ & & & 0.2642 \\
\hline 4 & $\varepsilon_{0}$ & & & & 0.3350 \\
\hline \multirow[t]{2}{*}{5} & & & & $\varepsilon_{0}$ & 0.3600 \\
\hline & $\varepsilon_{0}$ & $\varepsilon_{0}$ & $\varepsilon_{0}$ & $2 \varepsilon_{0}$ & - \\
\hline
\end{tabular}

7.2. Effect of $T_{y}$ on SINR and MI. When $T_{y}=0.01$, the energy distribution of transmitted waveform calculated based on SINR criterion and MI criterion and the resulting mutual information comparison are shown in Figure 11.

It can be seen that in the three cases, the mutual information is all largest based on MI criterion.

However, when $T_{y}=1$, the transmitted waveform energy distribution based on SINR criterion and MI criterion is obtained, and the resulting mutual information comparison is shown in Figure 12.

At this time, the mutual information obtained based on the SINR criterion is the largest.

For signal-to-interference-plus-noise ratio, the comparison based on SINR and MI criteria is similar.

It can be inferred from Figures 13 and 14 that when $T_{y}$ is large, the SINR criterion is better than the MI criterion, but when $T_{y}$ is small, the MI criterion is better than the SINR criterion, in which, for signal to interference noise ratio, SINR is better than MI in the most unfavorable case.

For this, we select one of the three cases and allocate certain energy to verify the influence of the size of $T_{y}$ on SINR and MI. For example, we select the robust transmitted waveform under the most unfavorable condition and allocate 5 units of energy.

Since the formula of SINR is independent of $T_{y}$, the value of SINR is constant with respect to the independent variable $T_{y}$ based on the SINR criterion. From Figure 15, it can be seen that the SINR criterion is always better than the MI criterion at the most worst case, while in the other two cases, the SINR criterion is better when $T_{y}$ is large, and the MI criterion is better when $T_{y}$ is small. At this time, there is a critical value of $T_{y}$. In the parameters assumed in this paper, the critical value is around 0.1 . 

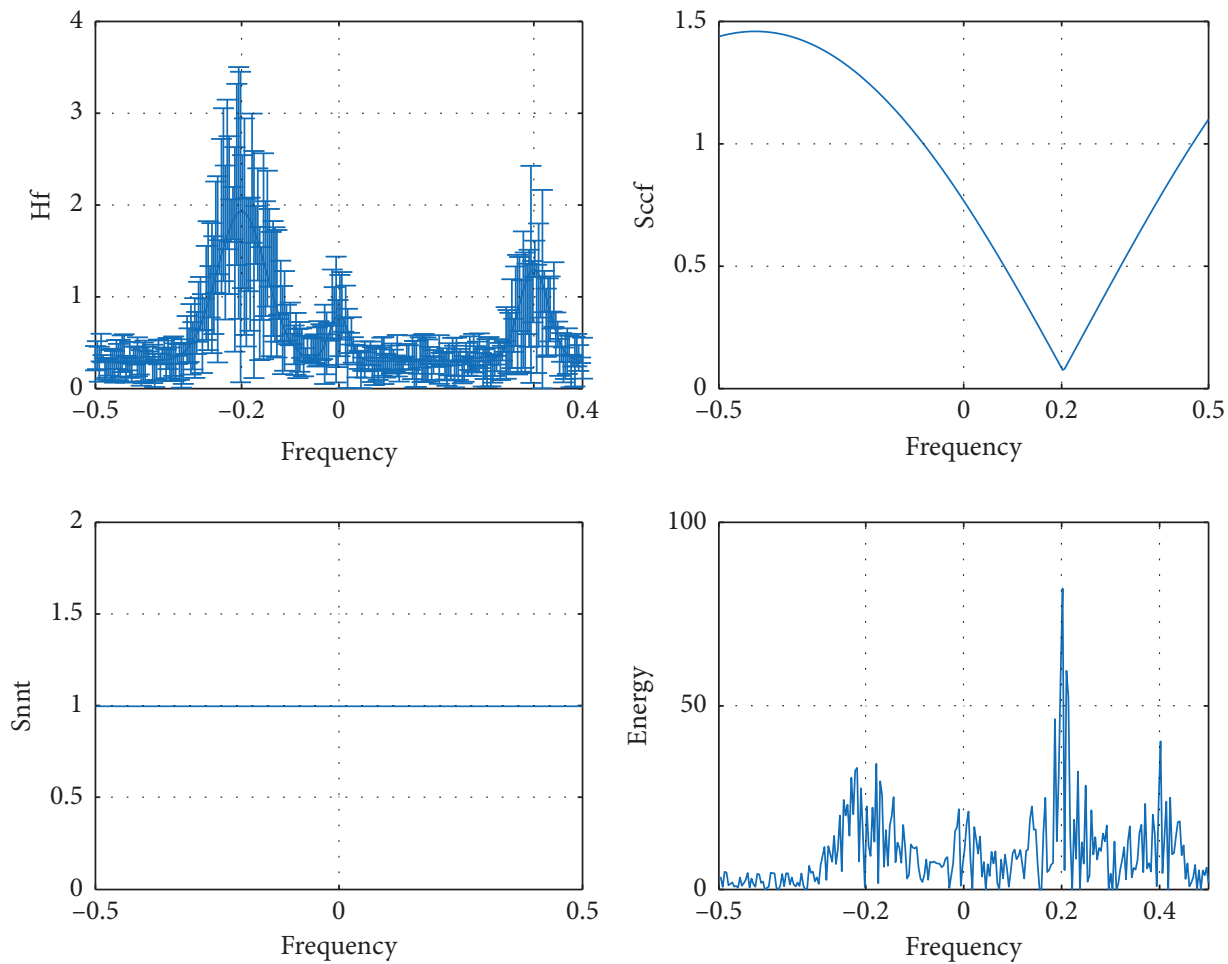

Figure 4: (a) Target spectrum, (b) clutter spectrum, (c) noise spectrum, and (d) transmitted waveform spectrum.

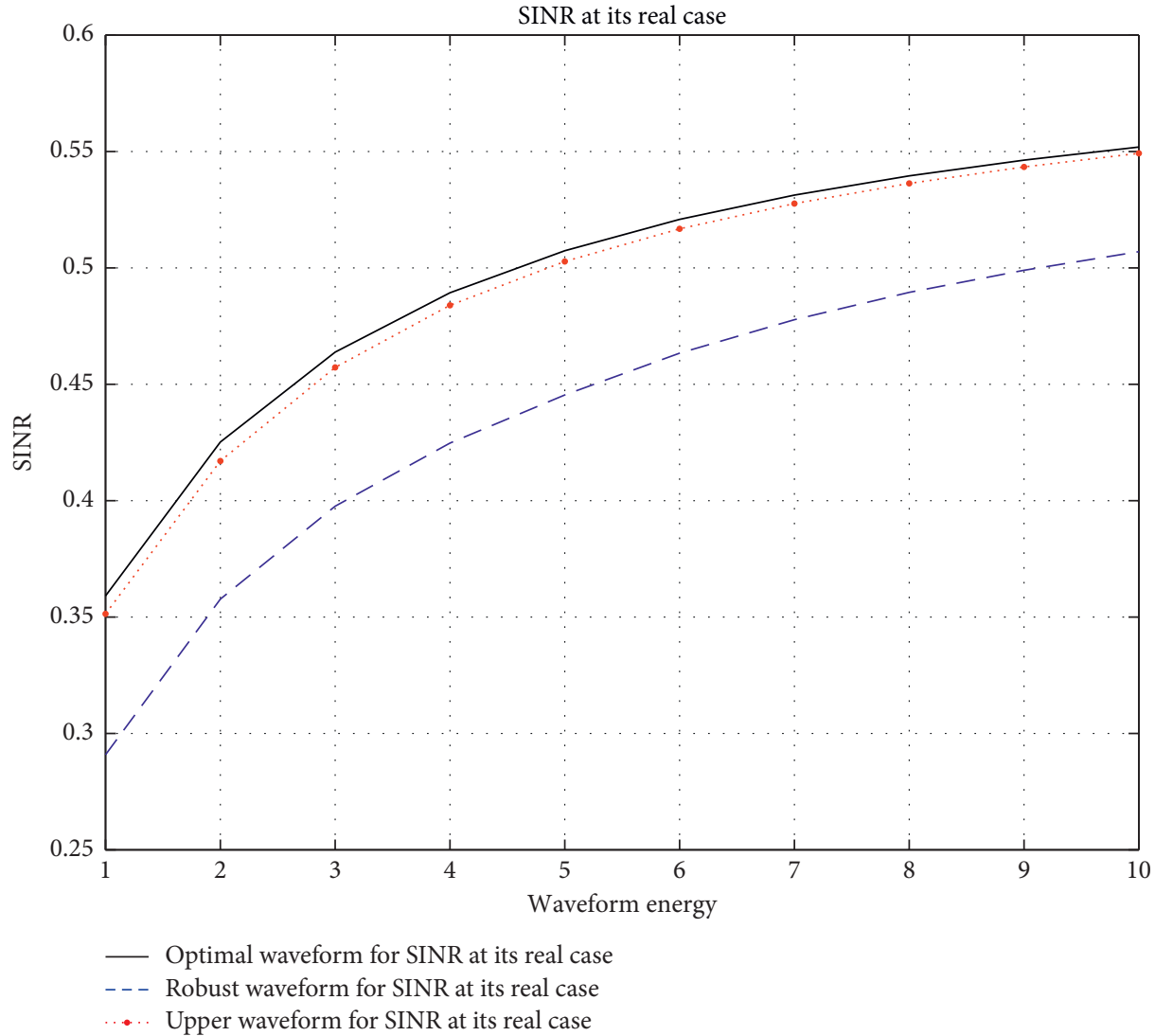

FIGURE 5: SINR of three waveforms when the real target spectrum is known. 


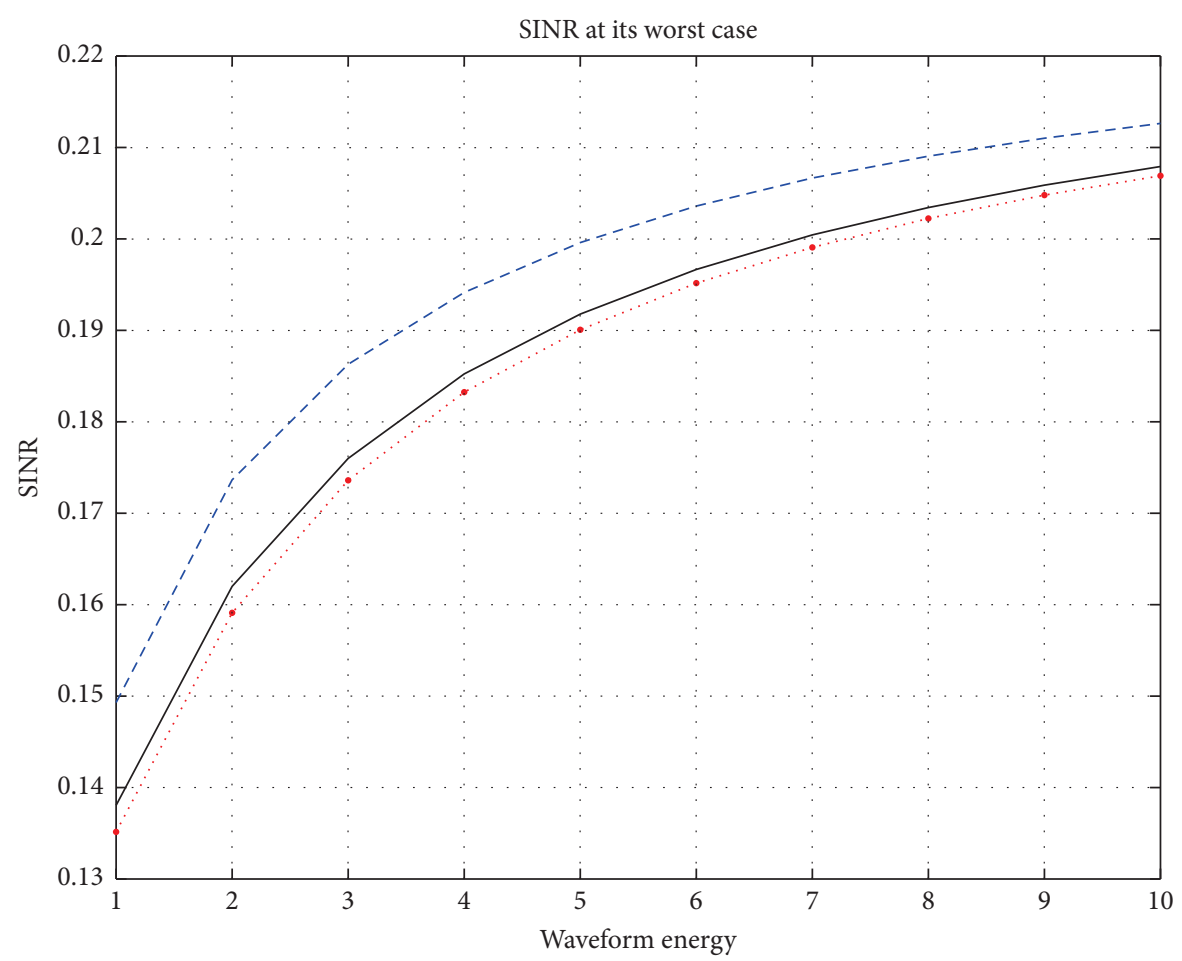

- Optimal waveform for SINR at its worst case

- - - Robust waveform for SINR at its worst case

... Upper waveform for SINR at its worst case

FIGURE 6: SINR of the three waveforms under the most unfavorable condition.

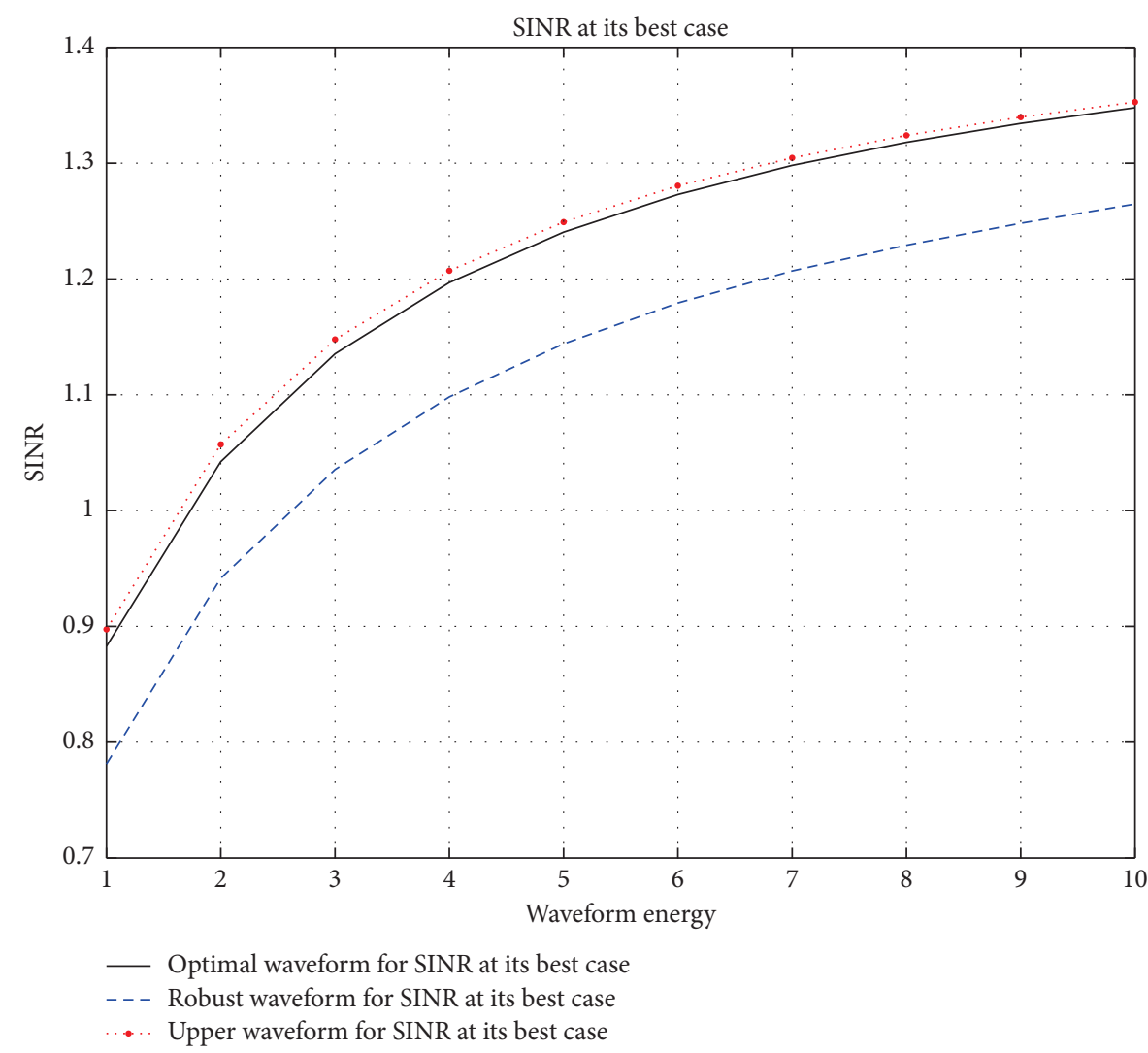

FIgURE 7: SINR of the three waveforms under the most favorable condition. 


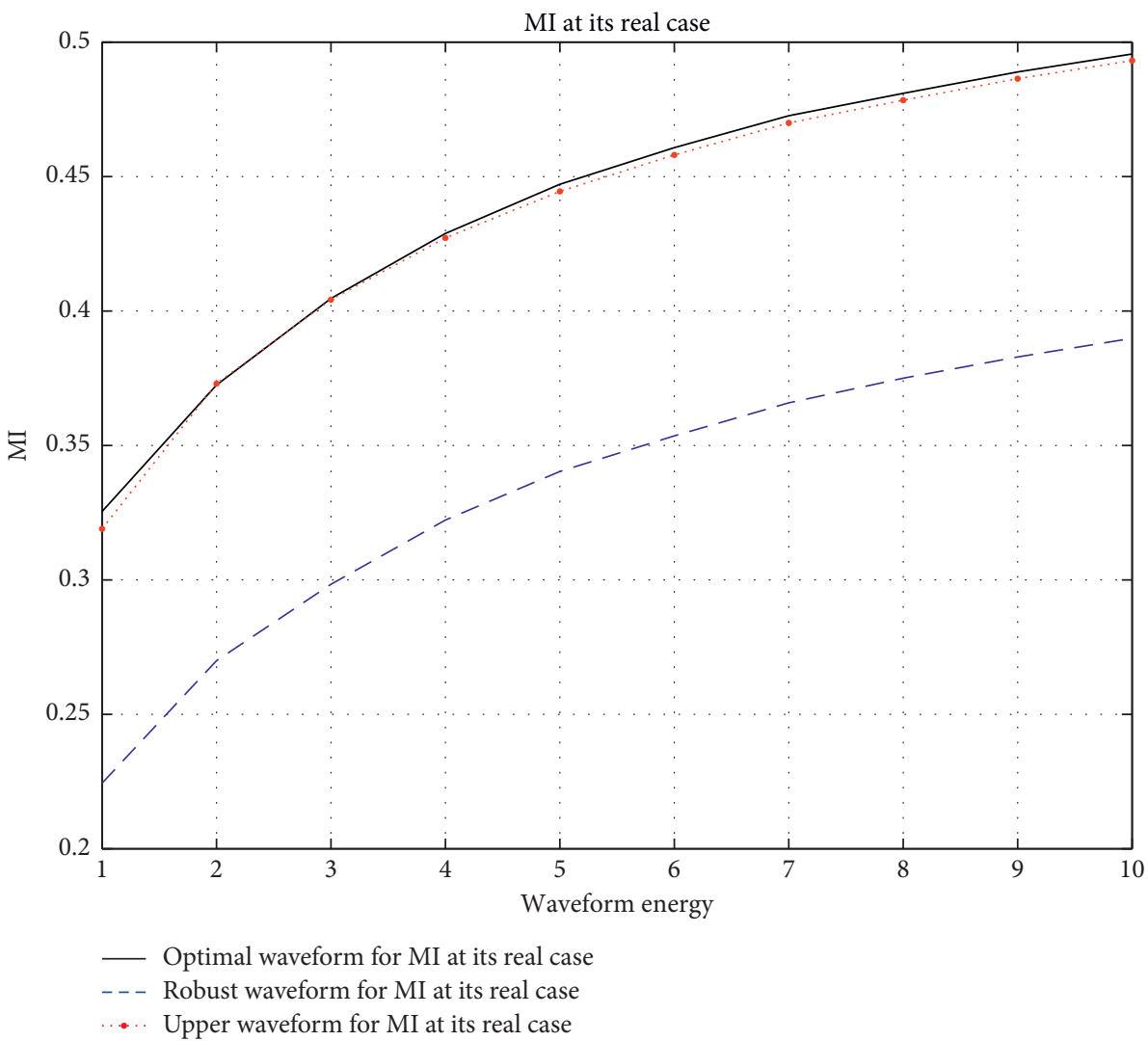

Figure 8: MI of the three waveforms when the real target spectrum is known.

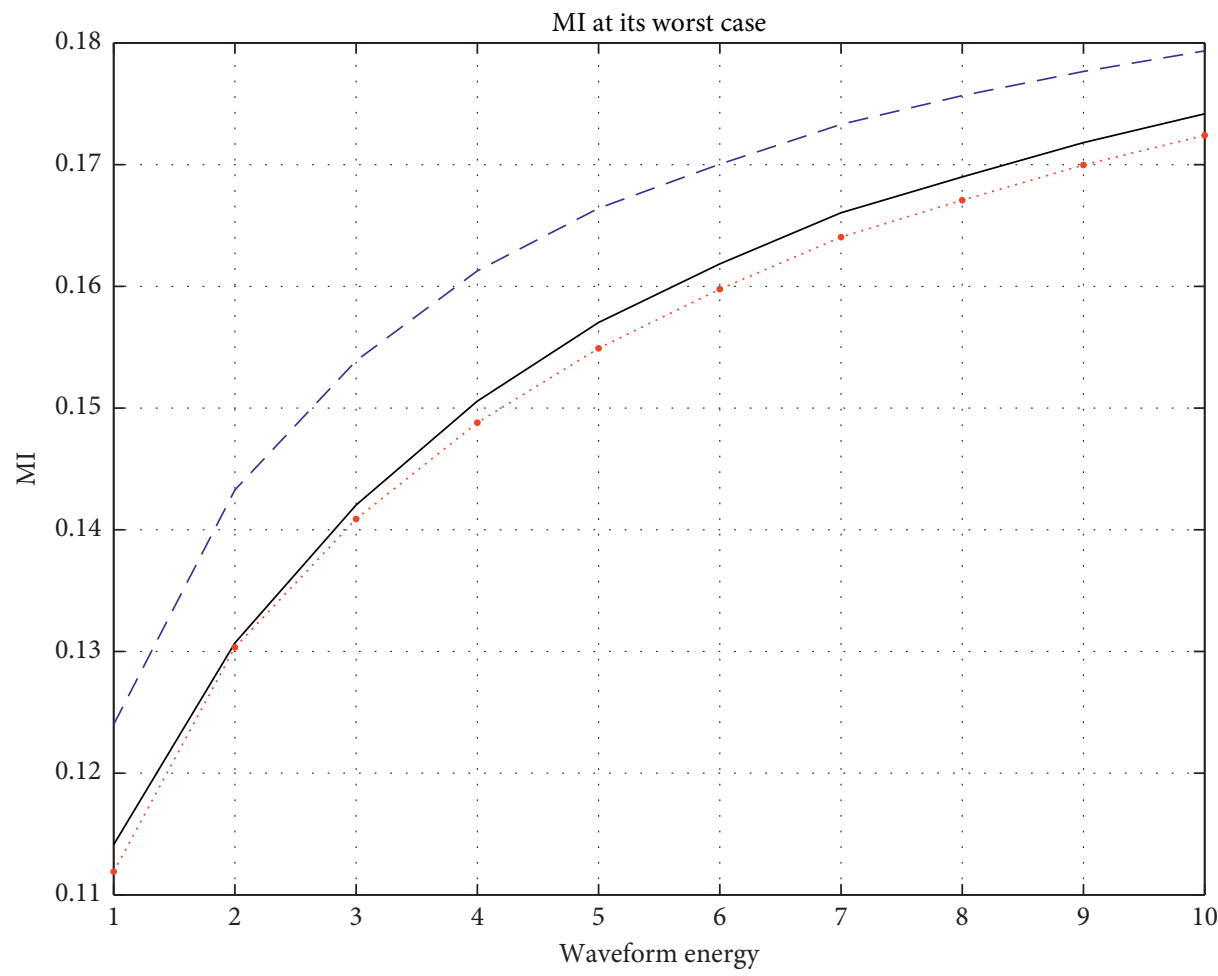

- Optimal waveform for MI at its worst case

- - - Robust waveform for MI at its worst case

... Upper waveform for MI at its worst case

Figure 9: MI of the three waveforms under the most unfavorable condition. 


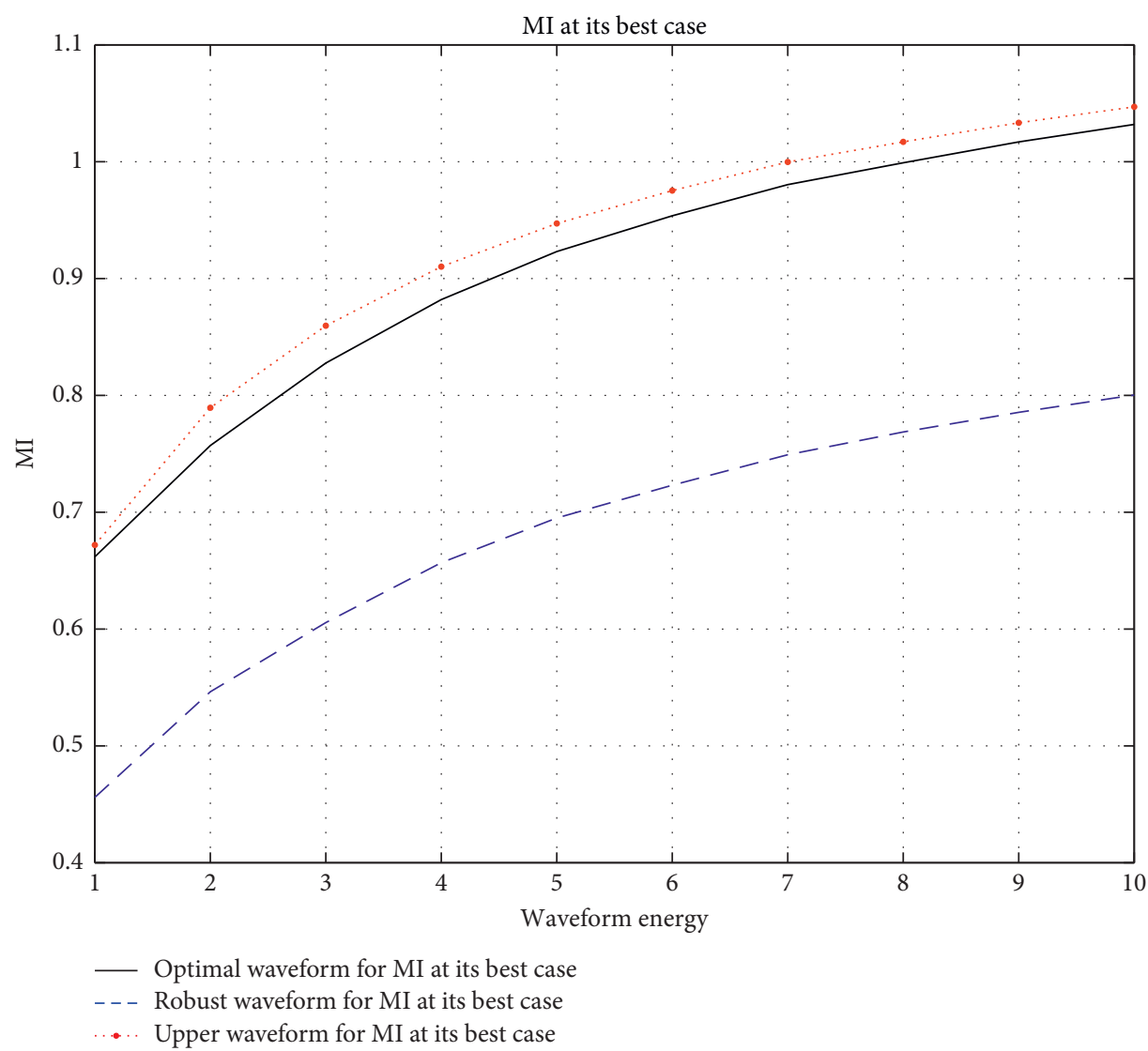

FIGURE 10: MI of the three waveforms under the most favorable condition.

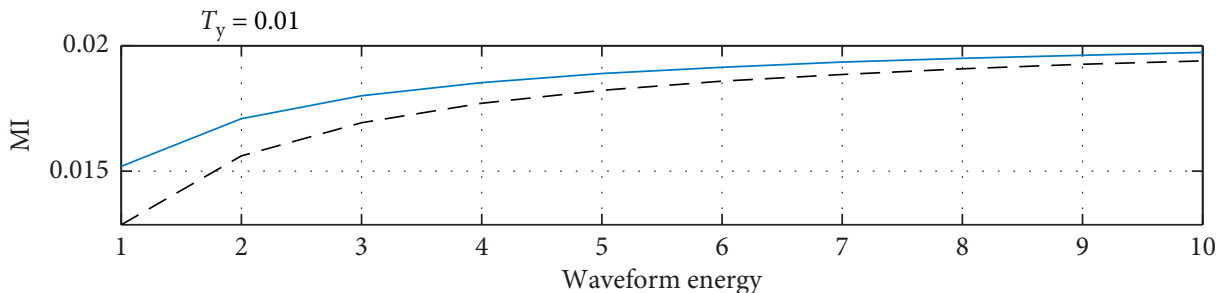

- - - Robust waveform for SINR at its worst case

_ Robust waveform for MI at its worst case

(a)

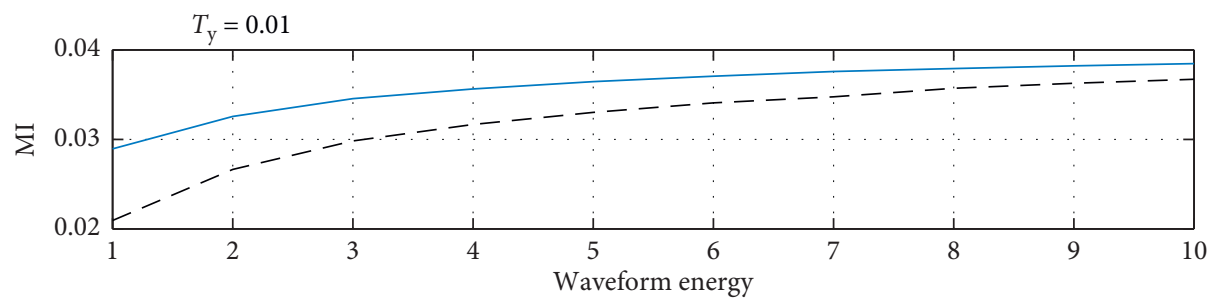

- - Robust waveform for SINR at its best case

Robust waveform for MI at its best case

(b)

FIgURE 11: Continued. 


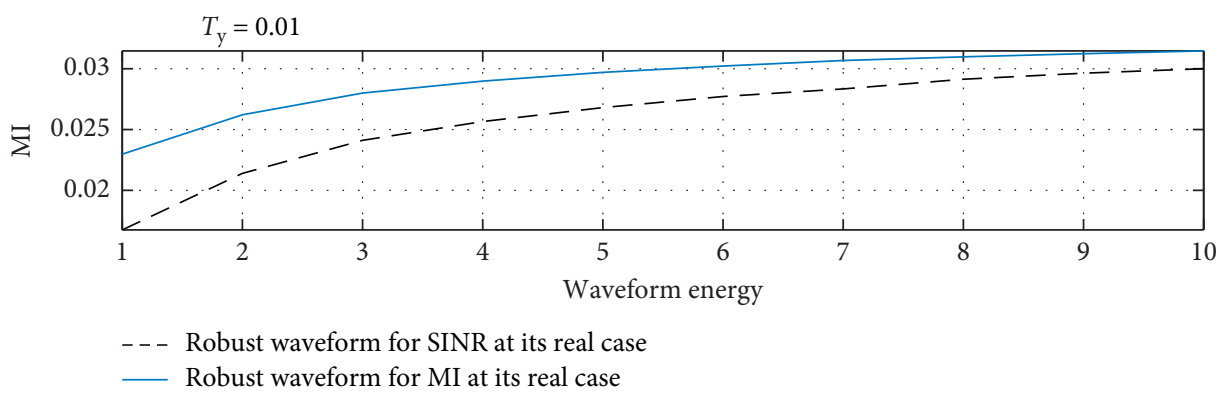

(c)

Figure 11: Comparison of MI under three conditions when $T_{y}=0.01$. (a) Comparison of MI at its worst case. (b) Comparison of MI at its best case. (c) Comparison of MI at its real case.

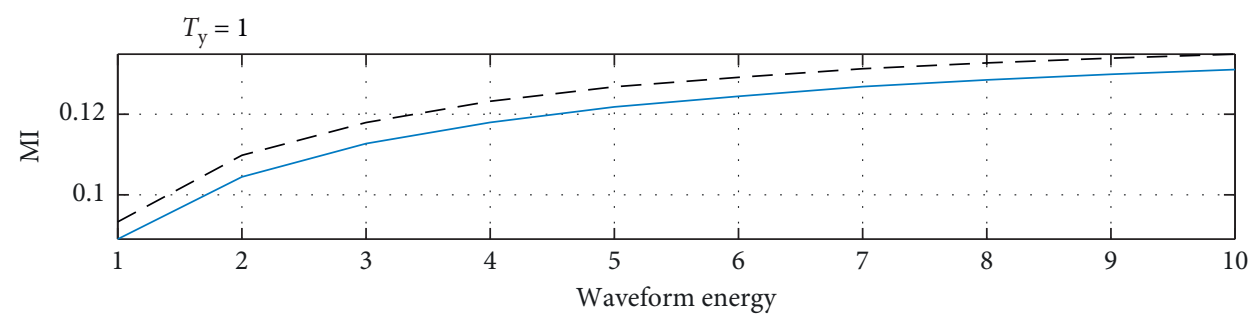

- - - Robust waveform for SINR at its worst case

_ Robust waveform for MI at its worst case

(a)

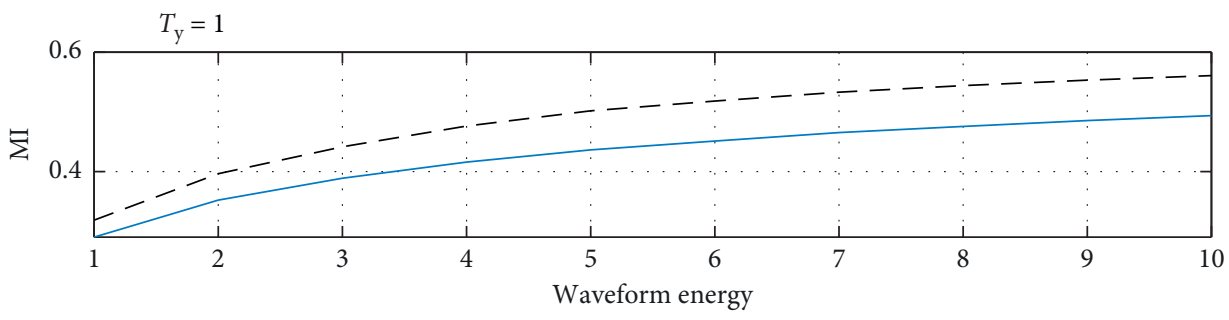

- - - Robust waveform for SINR at its best case

Robust waveform for MI at its best case

(b)

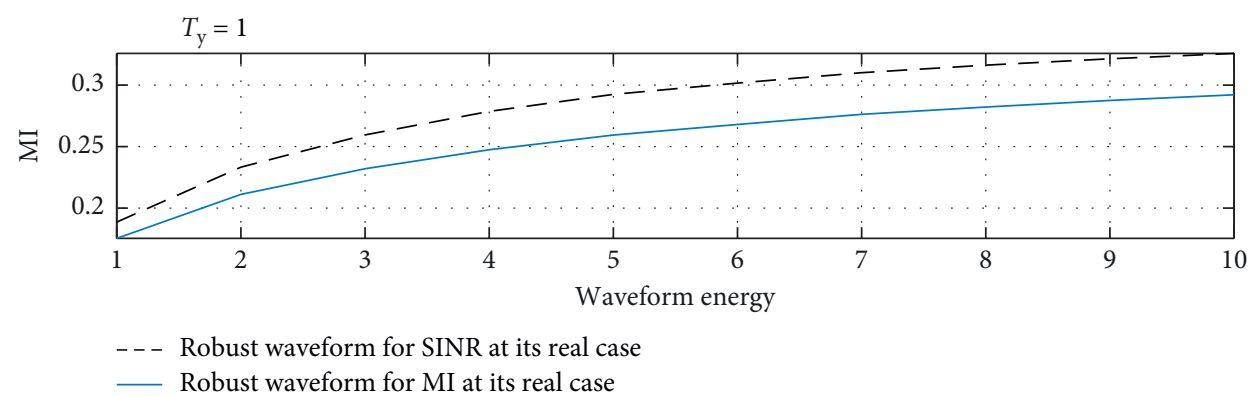

(c)

Figure 12: Comparison of MI under three conditions when $T_{y}=1$. (a) Comparison of MI at its worst case. (b) Comparison of MI at its best case. (c) Comparison of MI at its real case.

According to Figures 16 and 17, in three cases, when $T_{y}$ is large, it is better based on SINR criterion, and when $T_{y}$ is small, it is better based on MI criterion. At this time, the critical value is still around 0.1 .
7.3. Comparison of Two Water-Filling Algorithms and MMA. In the case of fixed energy constraint of 10 and $P=1000$ in MMA algorithm, the energy distribution and SINR of the transmitted waveform obtained by the three methods are shown in Figures 18 and 19. 


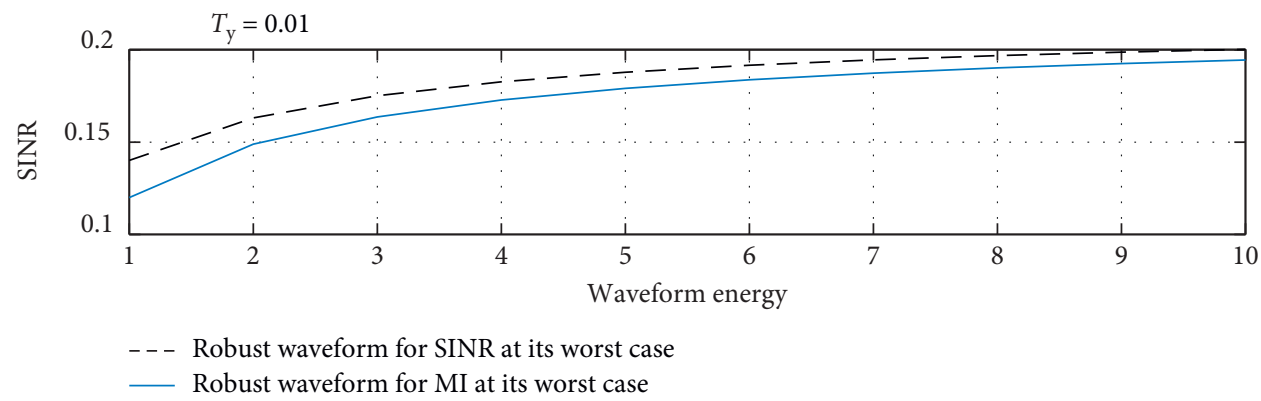

(a)

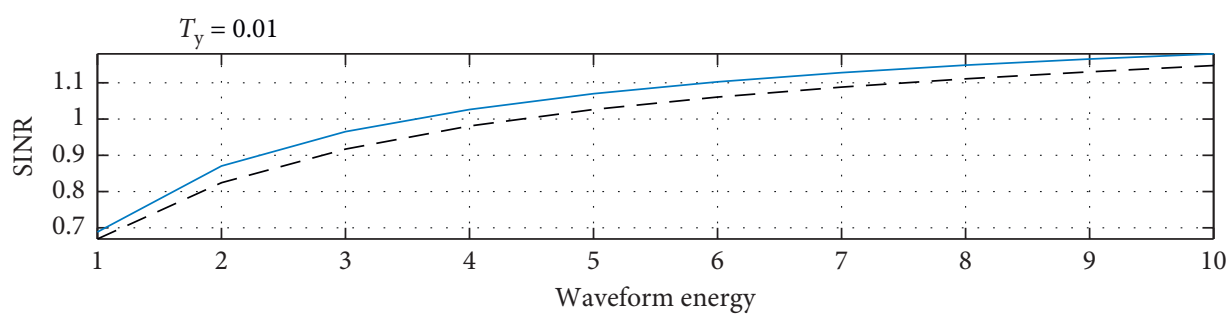

- - - Robust waveform for SINR at its best case

_ Robust waveform for MI at its best case

(b)

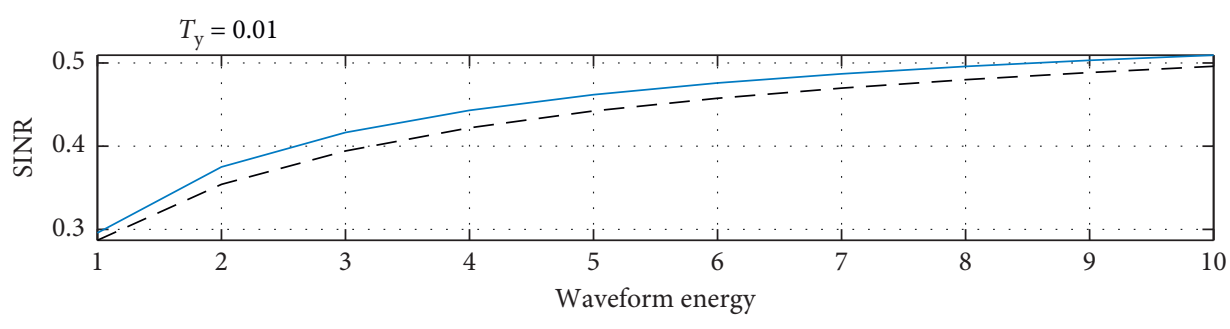

- - Robust waveform for SINR at its real case

_ Robust waveform for MI at its real case

(c)

FIGURE 13: Comparison of SINR under three conditions when $T_{y}=0.01$. (a) Comparison of SINR at its worst case. (b) Comparison of SINR at its best case. (c) Comparison of SINR at its real case.

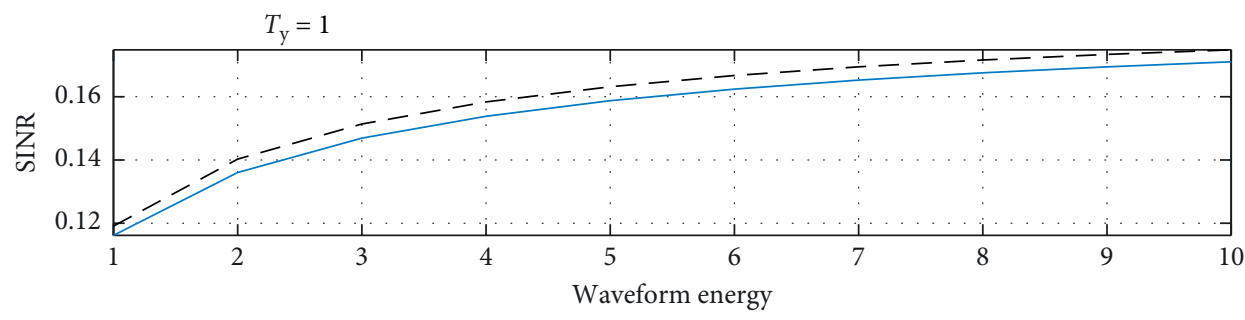

- - - Robust waveform for SINR at its worst case

Robust waveform for MI at its worst case

(a)

Figure 14: Continued. 


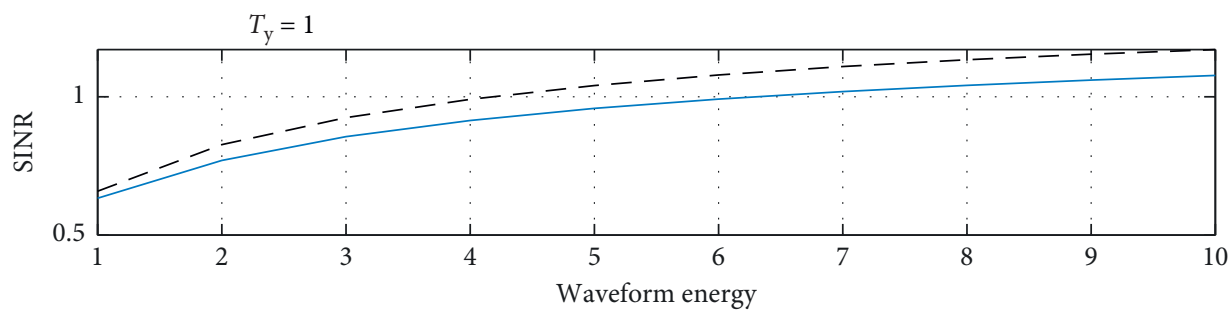

- - Robust waveform for SINR at its best case

Robust waveform for MI at its best case

(b)

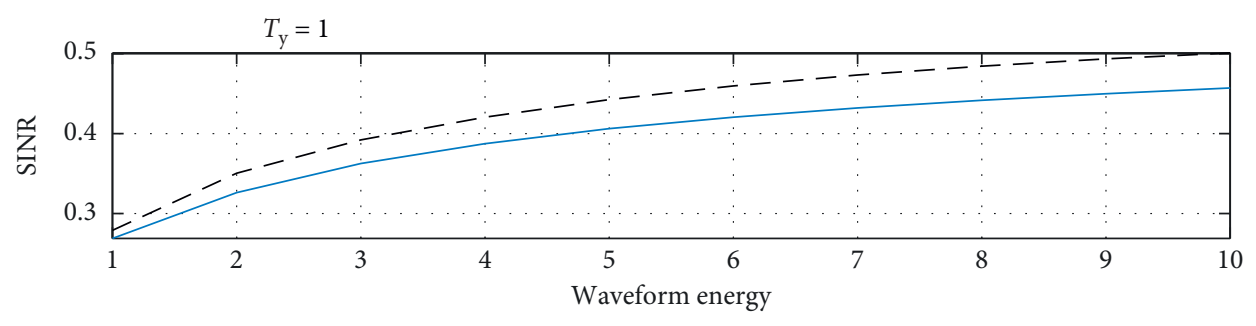

- - - Robust waveform for SINR at its real case

Robust waveform for MI at its real case

(c)

FIGURE 14: Comparison of SINR under three conditions when $T_{y}=1$. (a) Comparison of SINR at its worst case. (b) Comparison of SINR at its best case. (c) Comparison of SINR at its real case.

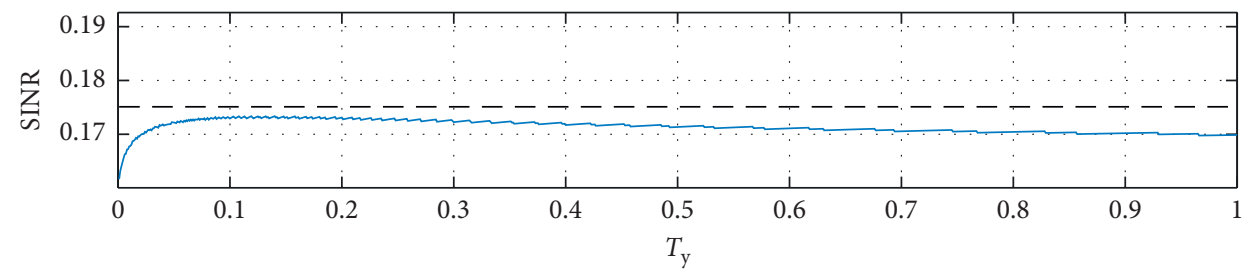

- - - Robust waveform for SINR at its worst case

_ Robust waveform for MI at its worst case

(a)

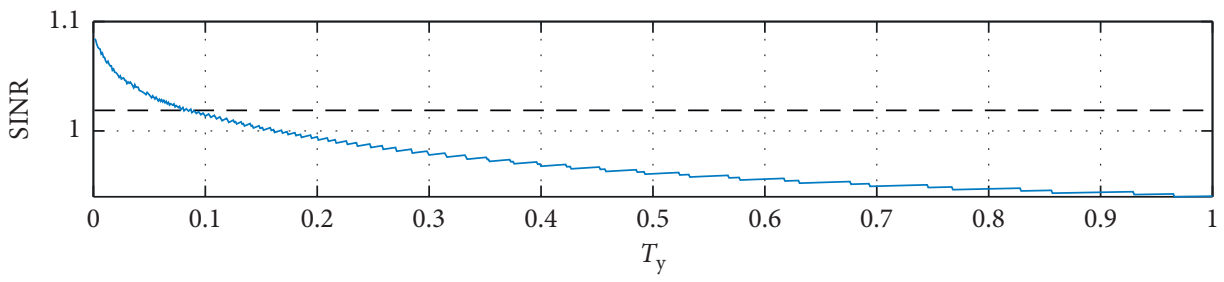

- - - Robust waveform for SINR at its best case

_ Robust waveform for MI at its best case

(b)

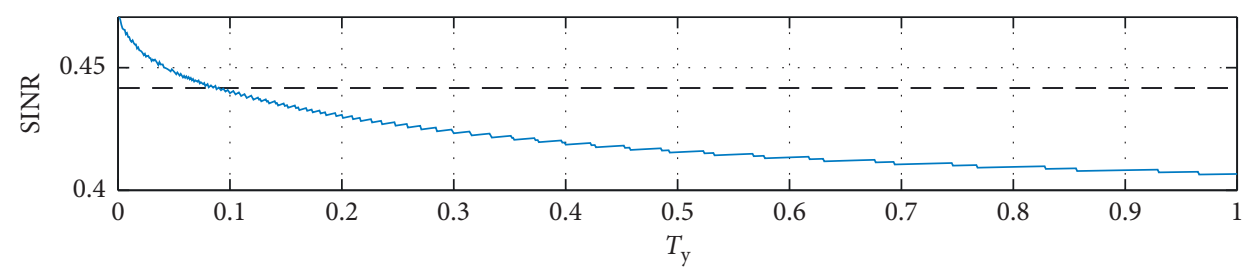

- - - Robust waveform for SINR at its real case

- Robust waveform for MI at its real case

(c)

FIGURE 15: Comparison of SINR under three conditions with the change of $T_{y}$. (a) Comparison of SINR at its worst case. (b) Comparison of SINR at its best case. (c) Comparison of SINR at its real case. 


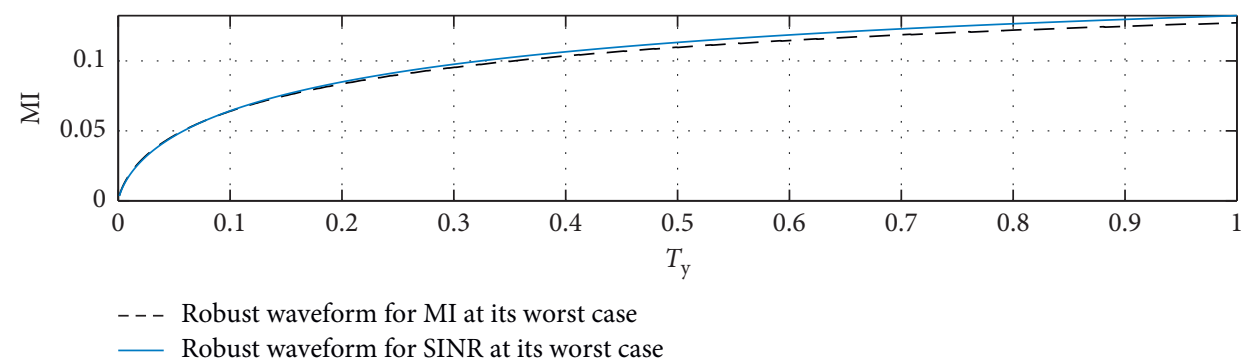

(a)

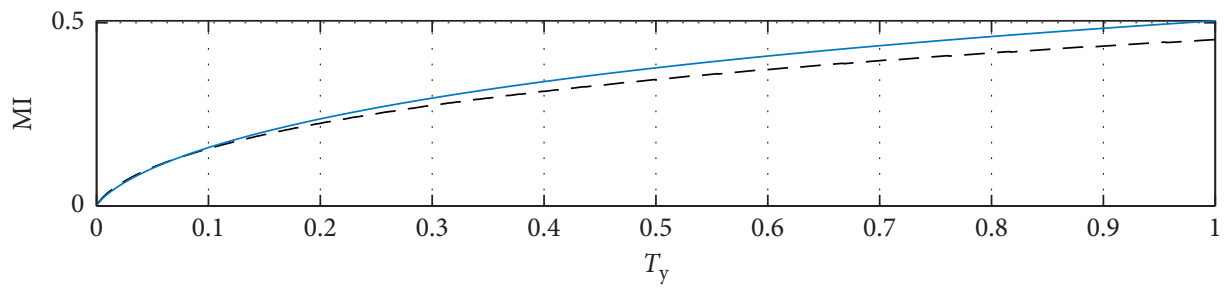

- - Robust waveform for MI at its best case

- Robust waveform for SINR at its best case

(b)

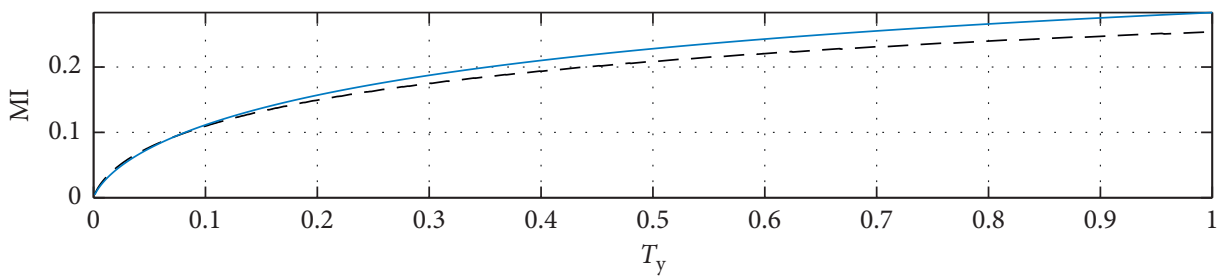

- - Robust waveform for MI at its real case

Robust waveform for SINR at its real case

(c)

FIGURE 16: Comparison of MI under three conditions with the change of $T_{y}$. (a) Comparison of MI at its worst case. (b) Comparison of MI at its best case. (c) Comparison of MI at its real case.

It can be seen from Figures 18 and 19 that the energy distribution of the transmitted waveform obtained by the three methods is very close, and the energy distribution at the frequency of $-0.2,0.2$, and 0.4 is relatively large. Moreover, the signal-to-interference-plus-noise ratio obtained by the three methods almost coincides with each other, so there is not much difference in the performance. In terms of simulation time, the MMA algorithm is greater than the general water-filling algorithm than the bisection waterfilling algorithm.

In the case that the fixed energy constraint is $10, T_{y}=10$, and $P=1000$ in MMA algorithm, the energy distribution and $\mathrm{MI}$ of the transmitted waveform obtained by the three methods are shown in the following figures.

It can be seen from Figures 20 and 21 that in terms of energy distribution of transmitted waveform, the two waterfilling algorithms distribute the energy more intensively around the frequency of -0.2 , while the MMA algorithm distributes the energy in a large number of frequency bands, mainly around $-0.2,0.2$, and 0.4 . Furthermore, the mutual information obtained by MMA algorithm is significantly larger than that obtained by the two water-filling algorithms. In terms of simulation time, the general water-filling algorithm is longer than the MMA algorithm than the Bisection water-filling algorithm. It can be seen that the use of bisection algorithm to search Lagrange multiplier improves the time performance, while the MMA algorithm is more effective in improving the mutual information between echo and target.

\section{Conclusions}

This paper studies the transmitted waveform design of cognitive radar. Cognitive radar can obtain information by interacting with the environment and improve the transmitted waveform through by sensing change of the surrounding environment. Most previous researches are based on known a priori information of target and environment. In this paper, considering the uncertainty of target in the real scenario, the robust waveform design technology based on signal-to-interference-plus-noise ratio and mutual information is adopted, and two methods to maximize the performance of MI and SINR are proposed. Firstly, the signal model is established, signal-to-interference-plus-noise ratio and the mutual information between the target and the echo are derived, 


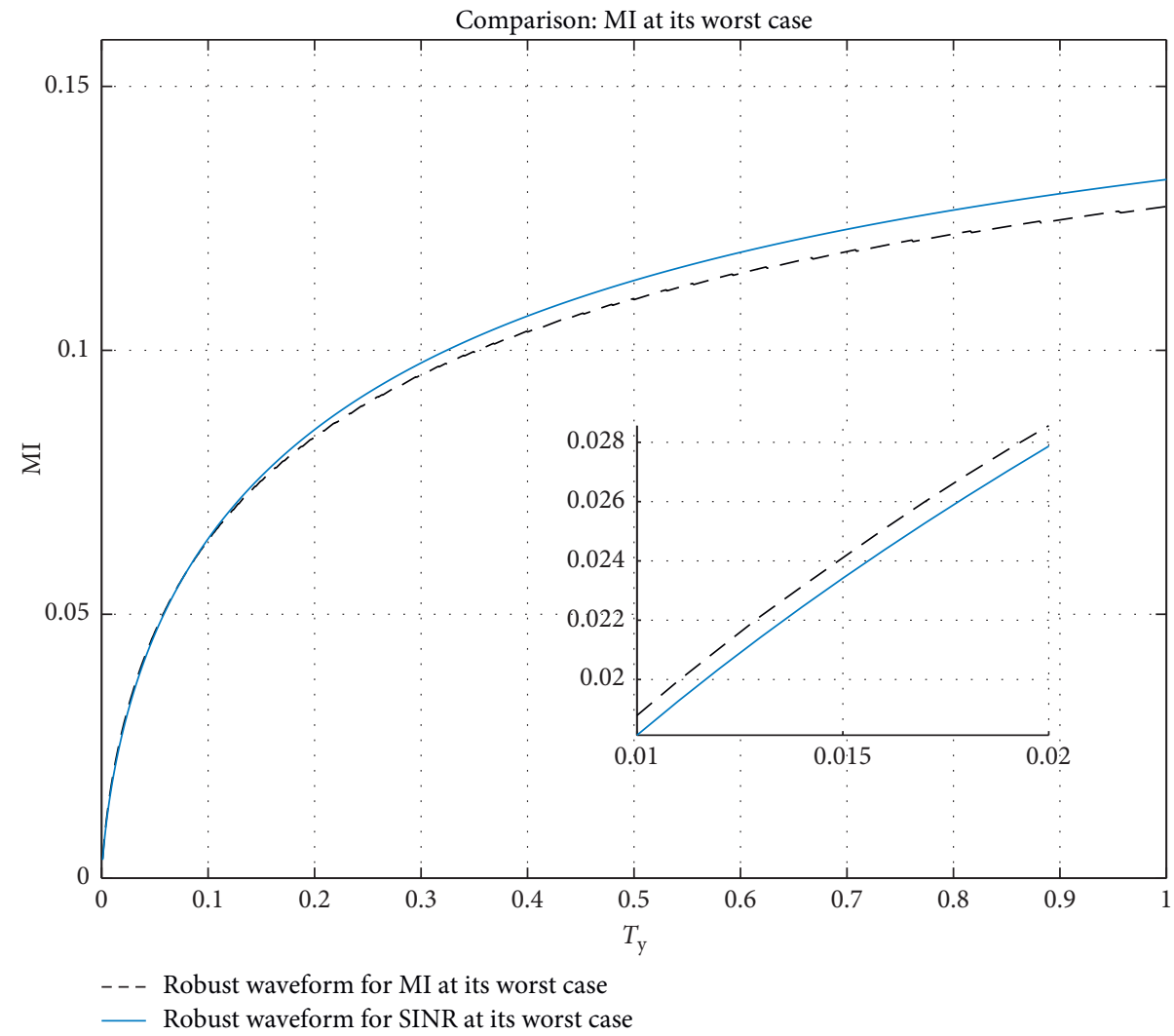

FIgURE 17: Comparison of MI under the most worst condition with the change of $T_{y}$.

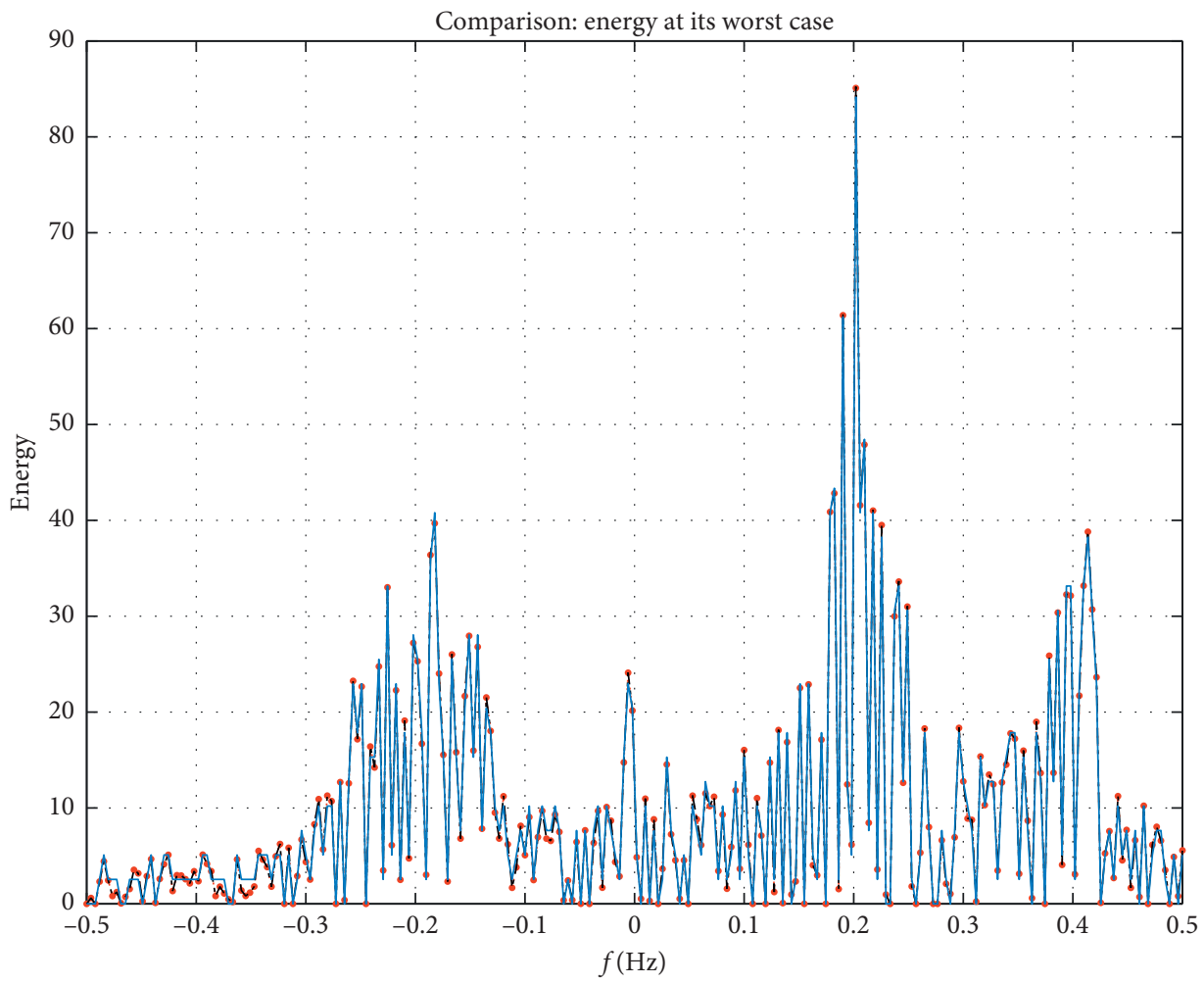

.... SINR of robust waveform by water-filling 1

. . - SINR of robust waveform by water-filling 2

— SINR of robust waveform by MMA

FIGURE 18: Energy distribution comparison of three SINR-based algorithms for transmitted waveform. 


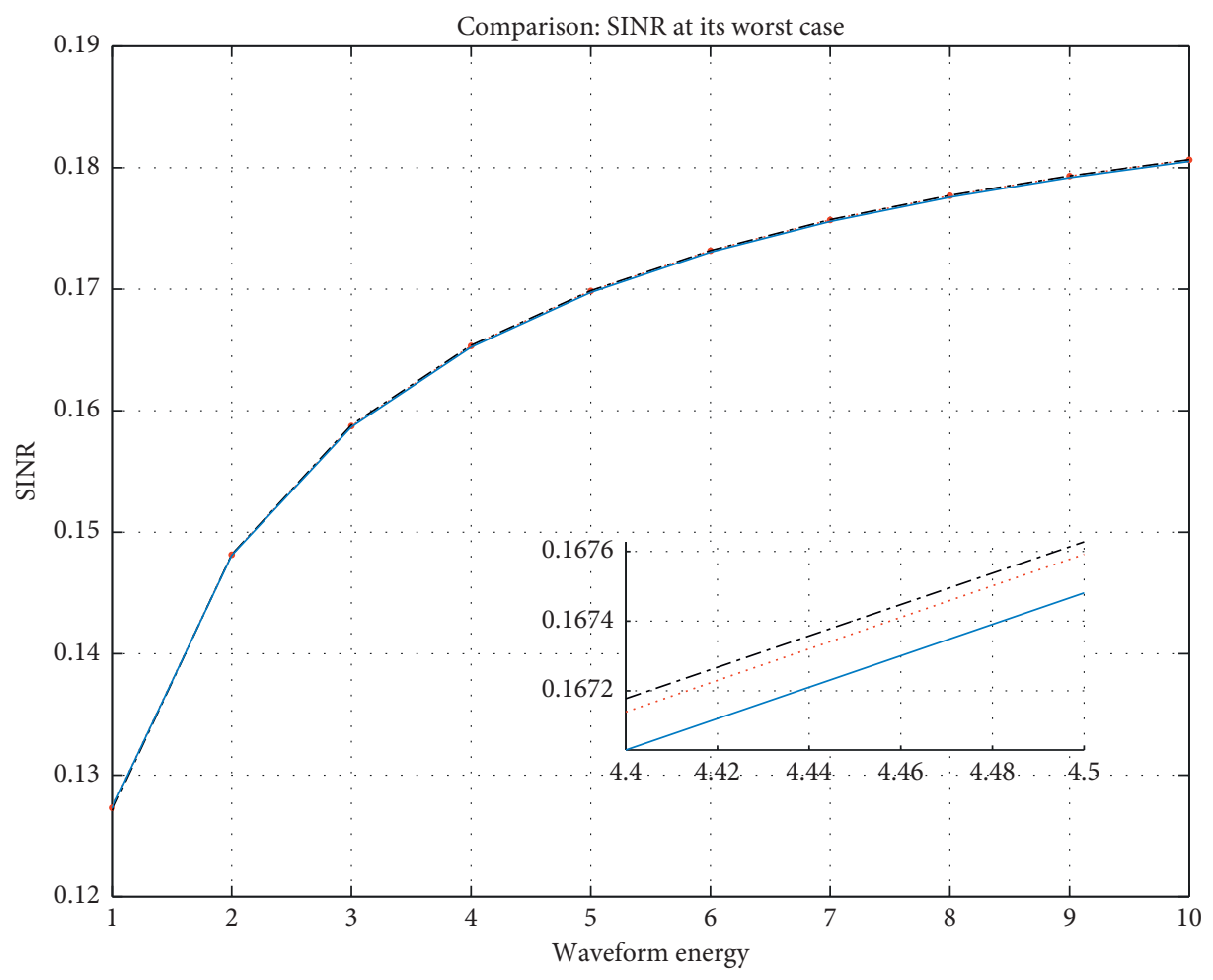

-... SINR of robust waveform by water-filling 1

-..- SINR of robust waveform by water-filling 2

— SINR of robust waveform by MMA

FIGURE 19: Comparison of SINR of three algorithms.

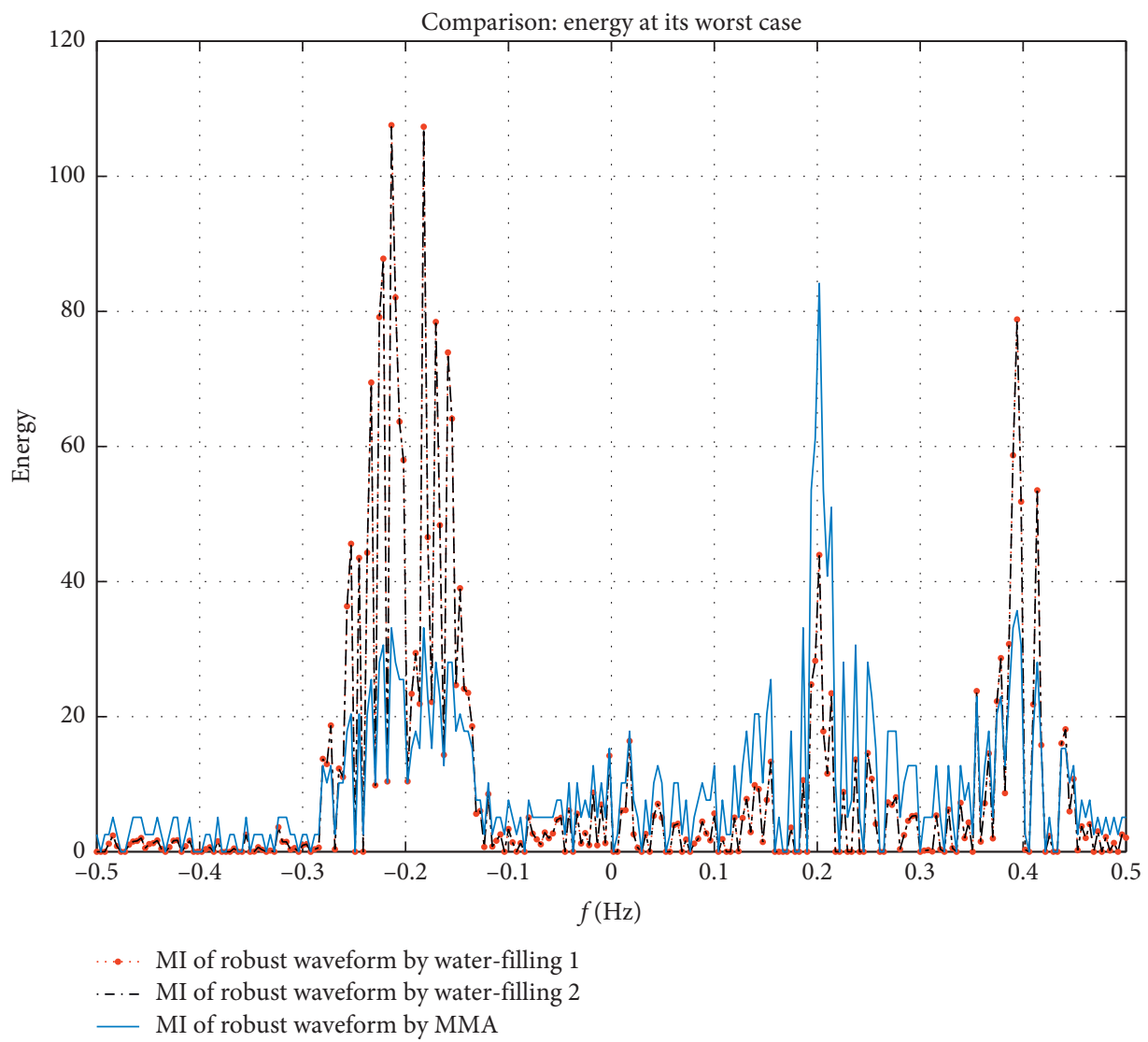

FIGURE 20: Energy distribution comparison of three MI-based algorithms for transmitted waveform. 


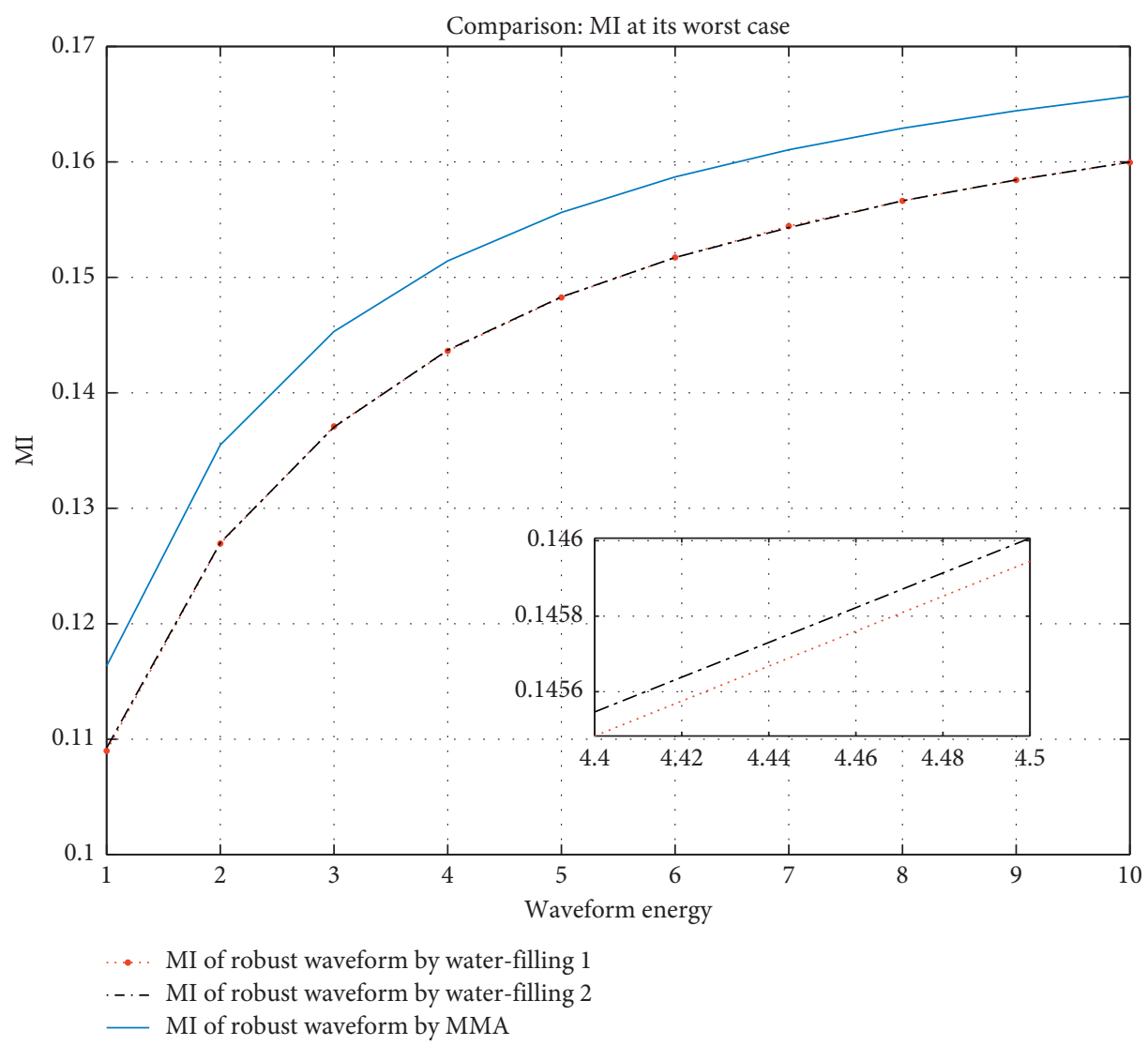

FIGURE 21: Comparison of MI of three algorithms.

and the robust signal model with upper and lower bounds is established. Then, based on the maximization of MI and SINR, two algorithms are proposed to solve the optimal robust transmitted waveform under the most unfavorable conditions. The traditional water-filling algorithm is improved and the time performance is promoted by using bisection algorithm when searching Lagrange multiplier. The maximum marginal allocation algorithm is introduced to maximize SINR and MI by selecting the optimal allocation of energy at each stage. Under known target spectrum, known target spectrum lower bound (worst case), and known target spectrum upper bound (best case), we simulate and compare the SINR and MI of several waveforms, respectively, and conclude that robust waveforms can improve the performance of the radar system in the most unfavorable situations. This paper also explores the effect of echo observation time on SINR and MI. Simulation results show that there is a critical value for the impact of echo observation time on transmitted waveforms based on different criteria. When the echo observation time is short, the MI-based transmitted waveform is better and when the echo observation time is long, the SINR-based transmitted waveform is more excellent. Finally, the advantages and disadvantages of MMA and two water-filling algorithms are compared, and the results show that the time performance of searching Lagrange multiplier is improved by using bisection algorithm, and the MMA algorithm is more effective in improving the mutual information between echo and target. In terms of signal-to-interference-plus-noise ratio, the time performance of the water-filling algorithm improved by bisection algorithm is better than that of MMA algorithm, but the SINR obtained by several algorithms has little difference. In terms of mutual information, the time performance of the water-filling algorithm improved by bisection algorithm is better than that of MMA algorithm, but MMA algorithm has the largest MI. In the future, we will deeply explore the principle of the influence of echo observation time on SINR and MI or optimize the time performance of MMA algorithm.

\section{Data Availability}

All data used to support the findings of this study are included within the article.

\section{Conflicts of Interest}

The authors declare that there are no conflicts of interest regarding the publication of this paper.

\section{Acknowledgments}

This work was supported by the Natural Science Foundation of Hebei Province (no. F2018501051). 


\section{References}

[1] S. Haykin, "Cognitive radar: a way of the future," IEEE Signal Processing Magazine, vol. 23, no. 1, pp. 30-40, 2006.

[2] M. R. Bell, "Information theory and radar waveform design," IEEE Transactions on Information Theory, vol. 39, no. 5, pp. 1578-1597, 1993.

[3] S. A. Kassam and H. V. Poor, "Robust techniques for signal processing: a survey," Proceedings of the IEEE, vol. 73, no. 3, pp. 433-481, 1985.

[4] B. Wang, X. Chen, S. Li, F. Xin, and J. Wang, "Robust waveform design based on jammer games in cognitive radar," in Proceedings of the 2018 10th International Conference on Communications, Circuits and Systems (ICCCAS), pp. 195198, Chengdu, China, December 2018.

[5] L. Wang, H. Wang, and M. Chen, "Cognitive radar waveform design for multiple targets based on information theory," in Proceedings of the 2016 CIE International Conference on Radar (RADAR), pp. 1-5, Guangzhou, China, October 2016.

[6] M. Hong and A. Garcia, "Averaged iterative water-filling algorithm: robustness and convergence," IEEE Transactions on Signal Processing, vol. 59, no. 5, pp. 2448-2454, 2011.

[7] X. Cheng, A. Aubry, D. Ciuonzo, A. De Maio, and X. Wang, "Robust waveform and filter bank design of polarimetric radar," IEEE Transactions on Aerospace and Electronic Systems, vol. 53, no. 1, pp. 370-384, 2017.

[8] R. A. Romero, J. Bae, and N. A. Goodman, "Theory and application of SNR and mutual information matched illumination waveforms," IEEE Transactions on Aerospace and Electronic Systems, vol. 47, no. 2, pp. 912-927, 2011.

[9] L. Wang, W. Zhu, Y. Zhang, Q. Liao, and J. Tang, "Multitarget detection and adaptive waveform design for cognitive MIMO radar," IEEE Sensors Journal, vol. 18, no. 24, pp. 9962-9970, 2018.

[10] Y. Liu, G. Liao, J. Xu, Z. Yang, and Y. Zhang, "Adaptive OFDM integrated radar and communications waveform design based on information theory," IEEE Communications Letters, vol. 21, no. 10, pp. 2174-2177, 2017.

[11] H. Wang, "Robust waveform design for MIMO-OFDM-based STAP in the presence of target uncertainty," IET Radar, Sonar \& Navigation, vol. 12, no. 9, pp. 1021-1027, 2018.

[12] G. Rossetti and S. Lambotharan, "Robust waveform design for multistatic cognitive radars," IEEE Access, vol. 6, pp. 74647475, 2018.

[13] L. Wang, Y. Zhang, Q. Liao, and J. Tang, "Robust waveform design for multi-target detection in cognitive MIMO radar," in Proceedings of the 2018 IEEE Radar Conference (RadarConf18), pp. 116-120, Oklahoma City, OK, USA, April 2018.

[14] W. Zhu and J. Tang, "Robust design of transmit waveform and receive filter for colocated MIMO radar," IEEE Signal Processing Letters, vol. 22, no. 11, pp. 2112-2116, 2015.

[15] Z. Rui, Z. Bo, L. Lin, Z. Xiaofen, and J. Bin, "Improved waveform design for non-Gaussian target classification in cognitive radar," in Proceedings of the IET International Radar Conference 2015, pp. 1-4, Hangzhou, China, October 2015. 